\title{
Le MUSiche di SCENA di Louis Pister Per La Tosca Di SARdoU: EDIZIONE CRITICA DELLA PARTITURA
}

\section{Abstract}

La tesi di dottorato di Erin M. Brooks, oggi Assistant Professor di Storia della musica presso la Crane School of Music della State University of New York at Potsdam, indaga il rapporto tra Sarah Bernhardt, prima interprete del ruolo di Floria in La Tosca, e la musica del suo tempo, compresa quella scritta appositamente per il teatro. All'interno di questo testo viene citata una rilevante scoperta dell'autrice, ossia un manoscritto delle musiche di scena composte da un certo Louis Pister per la pièce di Sardou, conservate oggi presso la National Library of Australia. Scopo di questo contributo è quello di proporre un'edizione critica delle musiche di scena composte da Pister, documento imprescindibile per l'analisi dell'opera di Sardou. Inoltre, si tenterà di ricostruire, attraverso lo spoglio dei periodici contemporanei, la ricezione del dramma $\mathrm{La}$ Tosca e il profilo biografico dello sconosciuto compositore della musica incidentale.

The PhD dissertation of Erin M. Brooks, now Assistant Professor of Music History at the Crane School of Music (State University of New York at Potsdam), investigates the relationship between Sarah Bernhardt, the first performer of Floria in La Tosca, and the music of her time, including incidental music. Within this text, a relevant discovery of the author is mentioned, namely a manuscript of the stage music composed by a certain Louis Pister for Sardou's pièce, preserved today at the National Library of Australia. The aim of this contribution is to propose a critical edition of the music composed by Pister, an essential document for the analysis of Sardou's play. Moreover, we will try to reconstruct, through the inspection of contemporary periodicals, the reception of La Tosca and the biographical profile of the forgotten composer of incidental music.

\section{INTRODUZIONE*}

Il 24 novembre 1887 il sipario del Théâtre de la Porte Saint-Martin si alza a mostrare agli spettatori l'interno della chiesa romana di Sant'Andrea della Valle: ha inizio la prima assoluta di La Tosca, il nuovo dramma di Victorien Sardou. La pièce diventerà presto uno dei più grandi successi di Sardou, autore già ben noto e apprezzato

\footnotetext{
* Questo contributo nasce come rielaborazione della prima parte della mia tesi di laurea magistrale in Scienze della musica e dello spettacolo, intitolata Musica e mise en scène nel teatro di parola: il caso della partitura di Louis Pister per La Tosca di Victorien Sardou, relatore E. Sala, correlatore D. Daolmi, Università degli Studi di Milano, a.a. 2019/2020. Ringrazio il prof. Roger Parker per i preziosi consigli in merito all'edizione, nonché il prof. Lucio Tufano, il quale mi ha segnalato il fatto che la gavotta del secondo atto non è una composizione originale di Pister, ma un'orchestrazione dell'ultimo movimento della dodicesima delle Sonate d'intavolatura, op. 2, di G. B. Martini.
} 
dal grande pubblico grazie ad alcune tragedie precedenti come Fédora del 1882 e Théodora del 1884.

Sebbene alcuni recensori contemporanei lo neghino, possiamo ascrivere La Tosca al genere del dramma storico: l'ambientazione nella Roma del 1800 non è un riferimento secondario o esornativo, ma un elemento per comprenderne l'apprezzamento del pubblico. In questi anni, il dramma storico è un genere particolarmente gradito, di cui Sardou è un rappresentante di primo piano:

Lo spettatore teatrale della Francia di fine Ottocento, e in particolare il fan di Sardou [...] al dramma chiedeva [...] di aderire al massimo alla verità storica del periodo in cui il drammaturgo aveva scelto di calare la vicenda, e pretendeva che quest'adesione venisse evidenziata. ${ }^{1}$

Il primo elemento per interpretare il successo di La Tosca è dunque la sua adesione a questo genere, caratterizzato dalla centralità dell'attendibilità storica dell'ambientazione: basta un'occhiata alle didascalie della pièce, ad esempio alla lunga e minuziosa descrizione della chiesa del primo atto o di palazzo Farnese nel secondo, per rendersi conto di quanto l'autore sia preciso nella resa estremamente dettagliata del panorama storico in cui la vicenda si svolge. A proposito di questa fondamentale caratteristica della mise en scène, occorre una precisazione: osservando attentamente le ambientazioni della pièce, ci si accorge di quanto l'attenzione dell'autore al dettaglio si scontri con numerosi errori sul piano architettonico e topografico. Come ricorda Viale Ferrero, è lo stesso Puccini, il quale ha grande considerazione per «la ricerca di una autenticità ambientale [che] gli serviva in quanto aveva significato», ${ }^{2}$ a criticare Sardou, in quanto ossessionato dai dettagli scenografici, ma in verità poco attento alla reale attendibilità degli stessi:

Stamani sono stato da Sardone per un'ora e circa al finale mi ha detto cose che non vanno [...] Nel farmi lo schizzo poi del panorama voleva che si vedesse il corso del Tevere passare fra S. Pietro e il castello!! io gli ho detto che il flumen passava dall'altra parte, sotto e lui tranquillo come un pesce ha detto oh questo è niente! bel tipo, tutto vita, fuoco e pieno di inesattezze storico-Topo-panoramiche. ${ }^{3}$

Le imprecisioni del dramma (evidenziate da Puccini in riferimento a Castel Sant'Angelo, ma riscontrabili anche in altri punti, come nell'identificazione della chiesa del primo atto) verranno dunque eliminate nell'allestimento del melodramma: «Il bozzetto messo in opera per la 'prima' al Teatro Costanzi $[\ldots]$ precisa $[\ldots]$ un criterio fondamentale dell'allestimento di Tosca: evitare le "inesattezze storico-topo-pa-

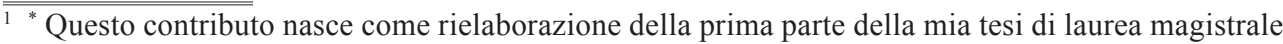
in Scienze della musica e dello spettacolo, intitolata «Musica e mise en scène nel teatro di parola: il caso della partitura di Louis Pister per La Tosca di Victorien Sardou», relatore Emilio Sala, correlatore Davide Daolmi, Università degli Studi di Milano, a.a. 2019/2020. Inoltre, ringrazio il prof. Roger Parker per i preziosi consigli in merito all'edizione.

SARDOU 2012, p. IX.

2 Viale Ferrero 2003, p. 45.

3 Biagi Ravenni - Schickling 2018, p. 272.
} 
noramiche" ma anche le citazioni precise e le ricostruzioni pedanti». ${ }^{4}$ Tali imprecisioni non devono però far immaginare una contraddizione con l'attrazione del pubblico francese dell'epoca per l'adesione alla “verità" della narrazione; 1'interesse di Sardou è infatti quello di inserire un gran numero di dettagli apparentemente attenti, ma in verità spesso sommari e poco precisi, con il fine di produrre un'idea di presunta attendibilità gradita allo spettatore forse non particolarmente cosciente della topografia e dell'architettura romana, come sottolinea Schickling quando sostiene che «Sardou era particolarmente appassionato di dettagli storici [...] Allo stesso tempo, gli era indifferente che tali dettagli [... ] fossero plausibili: per lui la cosa più importante era 1'effetto scenico». ${ }^{5}$ Per usare le parole di Barthes, ${ }^{6}$ lo scopo di Sardou è quello di produrre nel pubblico un effet de réel, senza però curarsi di quanto gli elementi inseriti nella pièce corrispondano alla realtà degli oggetti e delle ambientazioni che rappresentano, il che produce ai nostri occhi, come a quelli di Puccini, una contraddizione, che evidentemente non emerge agli occhi degli spettatori francesi, probabilmente desiderosi di vedere sul palco un' ambientazione che rimandi a una generica idea di "italianità". Si noterà più avanti, infatti, quanto alcuni critici contemporanei lodino l'abilità di Sardou nella resa dettagliata dell'ambientazione, senza in verità curarsi (per mancato interesse o per ignoranza) della misura in cui mise en scène sia realmente attenta, precisa o coerente.

Il secondo elemento che garantisce il successo de La Tosca è senza dubbio la collaborazione tra Sardou e la «stella assoluta del teatro francese», ${ }^{7}$ ovvero Sarah Bernhardt, che interpreta Floria fin dalla prima. La fama globale della voix d'or, unita alla celebrità di Sardou, porteranno La Tosca in una serie di tournées in patria e all'estero, che condurranno all'ultimo stadio del successo della tragedia, ovvero il melodramma pucciniano; è infatti noto che l'idea di mettere in musica l'opera venga a Puccini dopo aver visto, a Milano, Sarah Bernhardt impersonare Floria Tosca, come il compositore testimonia in una lettera del 7 maggio 1889 a Giulio Ricordi:

Dopo due o tre giorni di ozii campestri per riposarmi di tutte le strapazzate sofferte, mi accorgo che la volontà di lavorare invece d'essersene andata, ritorna più gagliarda di prima... penso alla Tosca! La scongiuro di far le pratiche necessarie per ottenere il permesso da Sardou, prima di abbandonare l'idea, cosa che mi dorrebbe moltissimo, poiché in questa Tosca vedo l'opera che ci vuole per me, non di proporzioni eccessive né come spettacolo decorativo né tale da dar luogo alla solita sovrabbondanza musicale. ${ }^{8}$

A proposito delle rappresentazioni in Italia del dramma, la critica sembra dare una chiara e indicativa prova di questo secondo elemento di successo: non è tanto il dram-

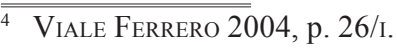

5 SChickling 2019, p. 70.

6 BARTHES 1988, pp. 152-158.

7 Sardou 2012, p. vi.

8 Biagi Ravenni - Schickling 2015, p. 101. Ibidem, nt. 1: «Puccini aveva assistito con Ferdinando Fontana a due rappresentazioni de La Tosca di Victorien Sardou, con Sarah Bernhardt nel ruolo eponimo, a Milano il 14 febbraio e a Torino il 17 marzo 1889, e, subito dopo la prima di Edgar, pensa alla prossima opera».
} 
ma di Sardou a venire apprezzato in sé, quanto la performance di Sarah Bernhardt. Possiamo leggere sulle pagine dell' Asmodeo che la rappresentazione al Carignano di Torino (del 17 marzo 1889, con Puccini tra il pubblico) «ebbe un successo immenso, malgrado i difetti del lavoro, grazie alla esecuzione sublime della Bernhardt, freneticamente applaudita», ${ }^{9}$ così come la lunga recensione su $L$ 'Indipendente spende molte lodi all' attrice, lasciando alle ultime righe la critica al drammaturgo: «Convien ringraziare Vittoriano Sardou, che annullando sé stesso per un giorno ha dato modo all'attrice di manifestarsi tutta qual è la sua individualità artistica gigantesca»». ${ }^{10} \mathrm{~L}$ 'articolo forse più indicativo in questo senso è la recensione apparsa sul Corriere della Sera della rappresentazione al Teatro dei Filodrammatici del 12 febbraio (Puccini vedrà la replica due giorni dopo): siamo davanti a una pesante stroncatura di Sardou, visto come null'altro che un mezzo per far risaltare la splendida abilità della Bernhardt. Il critico non usa mezzi termini:

Un dramma più di questo comicamente tragico, impudentemente inverosimile, vacuamente faragginoso, grossolanamente melodrammatico, è difficile immaginare. Sardou non vi ha messo che i difetti della sua decadenza, una morbosa avidità di effetti eccessivi, una impertinente sicurezza di riuscire coi più volgari artifici ad ingannare la buona fede del pubblico. Non caratteri, non analisi, non ricerca, non finezza. [...] Ma tutto ciò che importa? - Chi ha udito il dramma ieri sera? - La Tosca per sé non esiste; la Tosca è Sarah Bernhardt. ${ }^{11}$

Allo stesso modo, la recensione ${ }^{12}$ della rappresentazione milanese del 1895 lascia grande spazio alle lodi per la prima donna, quasi dimenticando di citare l'autore del dramma. È perciò evidente come il successo, certamente in Italia ma non soltanto, de La Tosca sia dovuto in particolar modo all'interprete della protagonista, più che al dramma in sé: si noti, a margine, come ciò debba evidentemente essere una chiave di lettura per l'analisi del melodramma pucciniano, in quanto il compositore è colpito da una tragedia pensata già in origine per dare grande risalto all'attrice principale, fautrice del suo stesso successo.

Per tornare alle prime rappresentazioni in patria, cercando nella stampa periodica francese è possibile trovare un consistente numero di recensioni al dramma di Sardou. Il 25 novembre 1887 Le Figaro $^{13}$ pubblica un lungo articolo in cui viene recensita la tragedia. Dopo una presentazione dell'autore, il critico Vitu si concentra sui tre personaggi principali paragonandoli, in quello che definisce un «rapprochement inévitable», ai tre protagonisti della Marion Delorme di Hugo. Tutti i personaggi, spiega il critico, sono inventati, ma un elemento interessante è la resa esatta del milieu storico in cui la trama è ambientata: la Roma del 1800, attorno alla quale ruotano personaggi come la regina Maria Carolina o lady Hamilton, ed eventi come la battaglia di Marengo. È

\footnotetext{
$\overline{9 \text { Asmodeo, } 20 \mathrm{mar}}$. 1889, p. 4/vi.

10 L'Indipendente, 30 gen. 1889, p. 3/r.

1 Corriere della Sera, 13 feb. 1889, p. 3/I-III.

12 Corriere della Sera, 8 ott. 1895, p. 3/II.

13 Le Figaro, 25 nov. 1887, pp. 1/vI-2/III.
} 
interessante il modo in cui Vitu descrive il barone Scarpia, per la creazione del quale Sardou, pur utilizzando la propria fantasia, non ha esagerato:

Le nom du policier Scarpia ne figure pas dans l'histoire; mais il en existe mille de son espèce, à la fois sanguinaires, libertins, hypocrites et lâches, ayant pour collaborateurs des grands seigneur spires que des brigands de grand chemin, et des brigands de grand chemin transformés en généraux, chamarrés d'ordres et de croix, tells que Michele Pezza, plus connu sous le sobriquet Populaire de Fra Diavolo, et Gaëtan il Mammone, qui s'abreuvait du sang humain recueilli tout chaud au sortir des veines de ses victimes palpitantes.

Sarah Bernhardt è descritta come graziosa nelle scene amorose del primo atto, ma capace di trasformarsi nel giro di poco tempo in una grande attrice tragica, soprattutto nel momento culminante del dramma, ossia la scena dell'uccisione di Scarpia, di cui viene lodata la misteriosa uscita di scena dell'artista. Pierre Berton è un magistrale Scarpia, che sa donare un'aura di gentiluomo al perverso barone, rendendolo un personaggio ancor più terrificante; di grande impatto è l'agonia dell'ufficiale dopo la pugnalata inferta da Floria, recitata in maniera straziante. Le scenografie, in particolare la chiesa di Sant'Andrea della Valle, palazzo Farnese e Castel Sant'Angelo, sono rese con quella che il critico interpreta come una rigorosa precisione storica: «Une représentation de la Tosca vaut un voyage à la Ville Éternelle».

Il giorno dopo due giornali pubblicano una recensione della tragedia: Le XIX $X^{\mathrm{e}}$ siècle e $L e$ Petit Parisien. L'articolo su Le XIX ${ }^{\mathrm{e}}$ siècle ${ }^{14}$ inizia ricordando la richiesta di Sardou di escludere la stampa dalla prova generale, enfatizzando le difficoltà che i giornalisti hanno di conseguenza incontrato nell'esposizione della trama di un'opera così importante; segue una descrizione particolareggiata della trama. Fouquier considera La Tosca «pour ce qu'elle est, c'est-à-dire un drame d'aventure»: se l'ambientazione storica è dettagliata, esatta e interessante, non è però possibile parlare di dramma storico, in quanto i fatti principali sono inventati e non ripresi dalla storia. Non si può parlare neanche di un dramma in cui sia centrale l'evoluzione dei personaggi, in quanto tutti gli attori interpretano una parte piuttosto fissa: Scarpia è un completo scellerato, Cavaradossi e Angelotti sono due eroi gioiosi, intrepidi e affascinanti, Tosca è una superstiziosa, passionale e amorosa italiana; una volta entrati in scena, non interessa a Sardou studiarne l'evoluzione psicologica. Le passioni sono un mezzo per arrivare al fine della tragedia, che è l'orrore tragico dell'avventura: tutto nel dramma è inserito per aumentare la sensazione di orrore. Nihil sub sole novum, secondo il critico, in quanto la situazione tragica è la stessa della Marion Delorme, sebbene la conclusione sia differente, e Scarpia non è altro che un grandioso Laffemas: l'originalità di Sardou non si vede tanto nella trama, ma nel movimento delle scene, nella verità (di nuovo, diremmo noi, solamente presunta) e vivacità dei tableaux, e soprattutto nella mise en scène. A tal proposito, vengono molto apprezzate le scenografie, curate nei minimi dettagli, soprattutto per quanto riguarda la ricca chiesa del primo atto, la villa di Mario, Castel Sant'Angelo e la sinistra ma splendida piattaforma da cui si getta Tosca. Interessante è il commento di Fouquier alla scena a palazzo Farnese, di cui il giornalista loda la mise en scène, a opera dello stesso Sardou, che riesce a unire commedia, opera e balletto, in uno «spectacle délicieux».

$\overline{{ }_{14} \text { Le XIX } X^{\mathrm{e}} \text { siècle, } 2} 6$ nov. 1887, p. $2 / \mathrm{II}-\mathrm{IV}$. 
La critica pubblicata su Le Petit Parisien,${ }^{15}$ come in altri casi, riassume la trama della pièce, e propone il paragone con la tragedia di Hugo Marion Delorme, che si traduce stavolta in una critica non molto leggera al drammaturgo. Parafrasando le parole dell'autore, Sardou avrebbe voluto tenere nascosta la propria opera, non ammettendo la stampa alla generale, in quanto sapeva di aver plagiato una tragedia precedente. Possiamo leggere tra le righe di questo articolo una vena di risentimento per l'esclusione dalle prove, che potrebbe aver spinto il giornalista, non del tutto disinteressato, a una certa faziosità che ne potrebbe aver deviato il giudizio. Anche il commento generale fornito dal critico è tutt'altro che positivo, soprattutto se paragonato alle recensioni fino a ora analizzate: «On avait essayé, par tous les moyens, de surexciter la curiosité autour de cette pièce: il faut bien reconnaître que la curiosité a été port déçue».

La recensione più lunga e più ricca di particolari è quella pubblicata il 28 novembre su Le Temps. ${ }^{16}$ Il più grande critico teatrale del tempo, Francisque Sarcey, sostiene che la pièce sia ben riuscita, e che potrebbe riuscire ancor meglio nei teatri d'oltreoceano. La descrizione della trama è accompagnata da alcuni interessanti elementi della scenografia, che è, come sappiamo, un fattore molto importante nei drammi storici. Nel primo atto, la bella e precisa decorazione della chiesa di Sant'Andrea rappresenta una calma statica che contrasterà presto con le passioni che si svolgeranno con quella scenografia. Sarcey descrive poi con toni entusiasti la scenografia del secondo atto, definendo Sardou un eccellente metteur en scène. Dalla fine del secondo atto, come evidenziano anche altri critici, inizia la vera tragedia, che Sarah Bernhardt sa interpretare magistralmente, passando da una cantante gaia a un'amante passionale e a tratti terrificante. La scena più spaventosa è quella del confronto tra Scarpia e Floria, che include la tortura di Cavaradossi e l'uccisione del barone: qui sta la vera abilità della Bernhardt, che sa far cambiare così velocemente l'atteggiamento di Tosca verso il nemico, prima pregandolo, poi adirandosi, poi gettandosi disperata ai suoi piedi; è alla fine di questa scena che gli applausi esplodono più fragorosi. Insomma, siamo di nuovo di fronte a una recensione che loda il binomio Sardou/Bernhardt.

Molto interessante è invece una recensione pubblicata su Le Figaro ${ }^{17}$ due anni dopo la prima: si tratta di una critica alla rappresentazione londinese del 1889, in cui $L a$ Tosca è andata in scena tradotta in inglese con alcuni cambiamenti della trama. Questa rappresentazione avviene al Garrick Theatre, a opera di Mayer, il quale aveva acquistato i diritti su La Tosca per il Regno Unito: è un successo, che verrà ripetuto per altre 300 volte a Londra, «une mine d'or pour M. Mayer». I cambiamenti nella trama vengono così descritti dal giornalista autore dell'articolo:

Comme il est indispensable de se plier au goût du pays et de ne pas froisser inutilement la pudeur exagérée, je ne le conteste pas, du pasteur anglais, les adaptateurs, MM. Grove et Hamilton, ont fait un changement dans le drame. La Tosca est mariée au comte Cavaradossi, mais elle est mariée clandestinement, ce qui modifie très peu la situation; ils ont supprimé les gémissements dans l'acte de la torture, e ils ont introduit un menuet dans celui du château

\footnotetext{
$\overline{15}$ Le Petit Parisien, 26 nov. 1887, p. 3/I-II.

16 Le Temps, 28 nov. 1887, pp. 1/I-2/vI.

17 Le Figaro, 4 dic. 1889, p. 3/II-IV.
} 
Saint-Ange. En résumé, MM. Grove et Hamilton se sont acquittés très habilement de leur tâche, en respectant l'œuvre de notre éminent écrivain, tout en faisant quelques très légères concessions aux désirs de la censure.

Il critico interpreta dunque i cambiamenti come piccoli e di scarsa importanza rispetto all'andamento della storia: in verità, la notizia dell'aggiunta di un minuetto nella scena a Castel Sant'Angelo non può certamente essere presa con tanta leggerezza. Purtroppo, non sono riuscito a trovare altre notizie su questo "minuetto inglese", che probabilmente si può collocare nel quarto atto, ossia nel confronto tra Tosca e Scarpia, prima che la donna uccida il barone. Considerando l'opera nel suo complesso, pare molto difficile leggere questo cambiamento come marginale, dato che l'atto della leggerezza e del bon ton dovrebbe essere il secondo, e soprattutto considerando che la scena del confronto tra Floria e Scarpia è descritta dai critici come il momento di massima tragicità del dramma, ed è quindi strano pensare a questa scena con l'aggiunta di un minuetto.

Per concludere la sezione sulle recensioni, è importante citare tre articoli scritti al di là della Manica, in un Regno Unito inizialmente restio ad accettare La Tosca sui palcoscenici dei propri teatri: si tratta di tre recensioni pubblicate sul settimanale londinese The Musical World. Bisogna notare come le prime due facciano riferimento al fiasco al Lyceum Theatre del luglio 1888. Il primo ${ }^{18}$ è un breve articolo di cronaca, in cui si annuncia la conversione in libretto di La Tosca, a opera di Fontana per Puccini, di cui il giornalista offre un'opinione tagliente:

There is no room in art for the bloody and repulsive class of plays to which this belongs. Not even the transcendent genius of a Bernhardt can make it tolerable; and it is to be hoped that Signor Puccini may be well advised enough to withdraw from an undertaking which can bring him no manner of credit.

Dato l'insuccesso dell'opera sul palcoscenico britannico, non ci si può stupire di una recensione negativa, anche se i toni sono particolarmente accesi e la stroncatura sembra essere totale. Il secondo articolo ${ }^{19}$ cerca di spiegare il motivo per cui, in Gran Bretagna, il dramma di Sardou non riesce a venire apprezzato; l'autore prende ad esempio la recitazione di Pierre Berton, che interpreta la parte di Scarpia:

The thing which struck me most in "La Tosca", after Madame Bernhardt's superb performance and the play itself, was the acting of M. Berton as Scarpia [...] I venture to say that such a piece of acting in an English drama would have been laughed or hissed off the stage on the first night. There was no attempt to copy nature in it all. The part was stagily written, and the actor treated it in a style of pure convention. He shouted and stamped and "took the stage" [...] Our actors try to be natural, to be "realistic"; there is their merit and their charm.

Una possibile spiegazione dell'insuccesso può essere perciò trovata nell'atteggiamento degli interpreti, i quali, a causa del gusto predominante nell'Europa continentale, tendono a recitare in una maniera che sembra simile a quella del grande attore, recita-

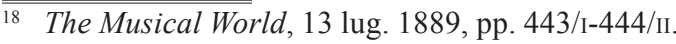

19 The Musical World, 27 lug. 1889, pp. 493/I-494/I.
} 
zione che invece in Inghilterra viene molto meno apprezzata rispetto al realismo. La recensione poi continua con una critica a Sardou, nella quale i toni tornano inclementi:

Sardou has followed the current, as usual, in this play. He is clever enough to give us stage-realism, now that he sees it is coming into fashion, just as he would have given us stage-romance if he had lived when Alfred de Musset was king. He drops his complexity, his clever intrigue; he does not set before us five acts of plot, but gives us five scenes of passion. We are supping full of horrors just now. Zola makes us sick with "La Terre", Ibsen makes us shudder with "Ghosts"; and Sardou will not be left out.

L'ultima recensione britannica ${ }^{20}$ riguarda la rappresentazione del 1889 tradotta in inglese. Non viene citato il misterioso minuetto che Le Figaro attesta come aggiunto al quarto atto, ma nel complesso la pièce sembra essere maggiormente apprezzata. Il punto più interessante è sicuramente il commento riguardo a Scarpia, interpretato da Forbes Robinson, il quale abbandona lo stile grandattorale per raggiungere un maggiore realismo, garantendo un certo successo alla serata. Nonostante la traduzione e il cambiamento dello stile di recitazione, l'apprezzamento della critica non è totale: l'unico pregio di La Tosca viene individuato nel dare la possibilità a un'attrice come la Beere (una Tosca addirittura migliore della Bernhardt) di farsi conoscere. È chiaro, in Inghilterra il dramma di Sardou non piace, nonostante il rifacimento:

I wrote to you last July about "La Tosca" at the Lyceum in French; I write you today about her in English at the Garrick; and then, I hope, no more of "La Tosca" for ever. Farewell, Italian singing-person who sings not: jump over your parapet into the eternal Nothing, and stay there!

Concludendo, da uno sguardo generale alle recensioni si può capire come La Tosca sia molto apprezzata in Patria, con l'eccezione di qualche critica, come quella su $L e$ Petit Parisien, che non è però indicativa in quanto determinata da una certa faziosità del giornalista. Abbiamo invece la conferma del fatto che, all'estero e soprattutto in Gran Bretagna, la pièce viene meno gradita dalla critica, la quale non si esime dal farlo presente con toni talvolta eccessivi e che possono rivelare una scarsa obiettività, in quanto il linguaggio teatrale e lo stile attoriale con cui viene messa in scena sono lontani dal gusto del momento, e anche a causa di un certo puritanesimo conducente a una difficoltà di accettazione di temi religiosi e legati a un concetto di pudore che in Francia è considerato troppo stringente.

\section{LOUIS PISTER}

Sulla partitura delle musiche di scena per la rappresentazione di La Tosca, individuata da Erin M. Brooks ${ }^{21}$ come anche sulle riduzioni per pianoforte del minuetto del secondo atto, si legge il nome del compositore: Louis Pister. A una prima occhiata, e anche in seguito a varie ricerche online, il suo nome potrebbe essere sostituito a quel-

\footnotetext{
20 The Musical World, 7 dic. 1889, pp. 867/II-868/II.

21 BRoOKs 2010, pp. 82-84.
} 
lo del filosofo Carneade nel celebre passo de I promessi sposi. Per trovare qualche informazione su Pister si rende necessario un ulteriore spoglio dei periodici, attraverso il quale è possibile ricostruire un minimo di biografia, soprattutto per quanto riguarda i suoi impieghi nel mondo dello spettacolo.

Grazie a una raccolta di vari documenti della segreteria del conservatorio di Parigi ${ }^{22}$ e al certificato di nascita conservato alle Archives Nationales de France, ${ }^{23}$ apprendiamo che Louis Jules Pister, figlio di André Pister e Léonie Lamotte, nasce a Givonne, un piccolo comune del dipartimento delle Ardenne, il 3 aprile 1846. Vincitore, a venticinque anni, del secondo premio in un concorso di armonia al Conservatorio di Parigi (notizia attestata anche sui periodici La France musicale ${ }^{24}$ e Le Ménestrel ${ }^{25}$ ), ricopre poi vari incarichi: è contrabbassista nell'orchestra dei Concerts Pasdeloup; professore di musica in alcune scuole di Parigi; maestro di cappella a Saint-Lambert; direttore d'orchestra al Théâtre de la Porte Saint-Martin, al Jardin d'acclimatation, al Casino de Royan e al Combat Naval.

Qualche anno dopo, Pister sembra aver iniziato una carriera come compositore. Le Ménestre ${ }^{26}$ pubblica un avviso per un concerto che si terrà, 117 dicembre 1884, presso il Cirque d'hiver, una sala situata al 110 di rue Amelot a Parigi: insieme ad altri brani, Benjamin Godard dirigerà anche il poema sinfonico Roland composto da Pister. Nel numero successivo ${ }^{27}$ del settimanale viene proposto un breve commento a questa composizione, rappresentata per la prima volta; il critico si esime dall'esprimere un'opinione sull'opera, citando la necessità di un secondo ascolto, che non tarda ad arrivare, in quanto Roland viene nuovamente rappresentato il 14 dicembre, come attestato in alcuni periodici. ${ }^{28}$ Oltre al commento su Le Ménestre ${ }^{29}$ della settimana successiva, in cui si afferma che «la légende Roland, de M. Louis Pister, ont fait une excellente impression», a seguito della seconda rappresentazione è possibile leggere una recensione a questa composizione, pubblicata sulla rubrica di cronaca musicale di Le Temps.${ }^{30}$ Veniamo a sapere che il poema sinfonico, prima opera di Pister a essere rappresentata, è composto a partire da una poesia in sei strofe di Paul Milliet, da cui il compositore ha tratto tre sezioni, intitolate Aude, Roncevaux e Apothéose. Secondo il critico, non è possibile ascrivere Roland al puro genere descrittivo, in quanto sono presenti alcuni momenti più evocativi, più vicini a una musica "assoluta". Si tratterebbe di un'opera composta a partire da una buona conoscenza dell'orchestra ma priva di idee originali: perciò Weber immagina che Pister sia più adatto, per il momento, come compositore di ouverture piuttosto che di poemi sinfonici di più ampio respiro.

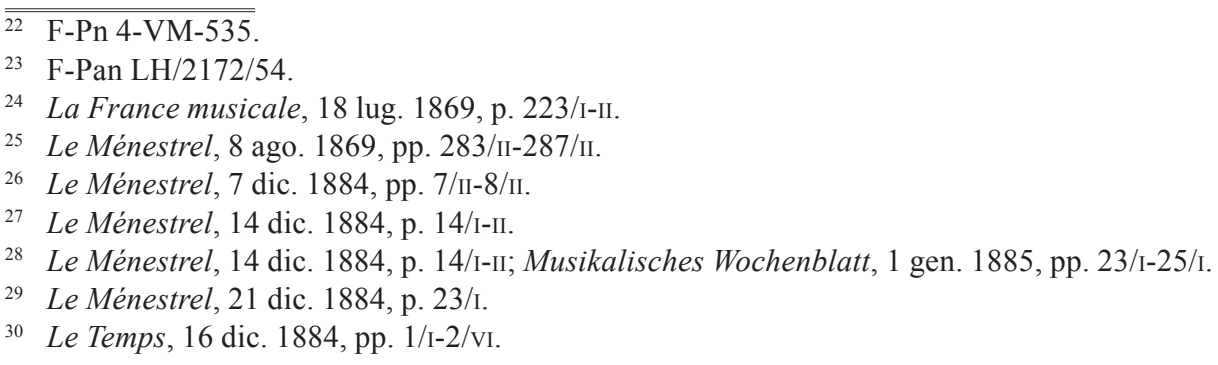


Sappiamo inoltre che il poema sinfonico verrà messo in scena nuovamente molti anni dopo, ${ }^{31}$ quando Pister sarà già direttore artistico del Jardin d'acclimatation: all'interno di un concerto del 10 maggio 1896 da lui stesso organizzato e diretto, il compositore inserisce Roland, che viene giudicato dalla critica come un'interessante pagina del direttore d'orchestra, ben accolta dal pubblico, brillantemente orchestrata e molto abilmente composta. Tornando ai primi anni di carriera del compositore, sappiamo da Le Ménestrel ${ }^{32}$ che nell'aprile 1885, sempre all'interno del ciclo dei Concerts modernes organizzato e diretto da Godard presso il Cirque d'hiver, viene rappresentata una Solitude di Pister, sulla quale però non abbiamo altre informazioni. L'ultima notizia sulle composizioni di Pister riguarda un concerto della pianista Louise Rückert del 1896: ${ }^{33}$ il programma prevede una serie di opere tra cui una composizione di Pister. Non sappiamo di quale brano si tratti, ma sappiamo che Pister non è dunque solamente un compositore per orchestra, ma si occupa anche di musica per pianoforte (che sia il minuetto del secondo atto di La Tosca, ridotto per pianoforte dallo stesso autore?).

Il primo impiego attestato di Pister come direttore d'orchestra è del 1890, al Théâtre de la Porte Saint-Martin, dove tre anni prima è andata in scena La Tosca. Il periodico La Rampe illustré $e^{34}$ ci offre un'immagine molto particolareggiata della sala dal punto di vista del palcoscenico, e ci informa del fatto che viene rappresentata la Jeanne d'Arc di Jules Barbier, con Sarah Bernhardt nella parte della Pulzella d'Orléans: l'orchestra e il coro sono diretti da Pister; apprendiamo inoltre dal periodico belga Le Guide musical $^{35}$ che la musica per il dramma è composta da Gounod. In effetti, come leggiamo sulle pagine di Le Ménestrel, ${ }^{36}$ Pister, già secondo direttore e amministratore dei Concerts Pasdeloup, è da qualche anno primo direttore d'orchestra del Théâtre de la Porte Saint-Martin, dopo esserne stato secondo direttore.

Dopo questa prima esperienza come direttore d'orchestra, nel $1893^{37}$ Pister ottiene l'incarico più testimoniato dalla stampa: è nominato direttore artistico del Jardin d'acclimatation, ovvero un parco zoologico situato in Bois de Boulogne, in cui la popolazione parigina ha la possibilità di assistere a dei concerti settimanali ospitati nel Palmarium, all'interno del Palais d'hiver. Una descrizione di questo luogo, utile per renderci conto degli spazi utilizzati e in generale di come siano pensati i concerti all'interno di questa sede, può essere trovata in uno studio di Pasler sui concerti popolari parigini:

The Jardin zoologique d'acclimatation opened in 1855 as a place to introduce, domesticate, and reproduce animals and plants from foreign lands. [...] After the Franco-Prussian war, when people were tired and needed places of leisure, the Jardin continued to encourage the study of natural history, but added recreation and amusement. To attract people to the park

\footnotetext{
$\overline{31}$ Le Ménestrel, 24 mag. 1896, p. 167/I.

32 Le Ménestrel, 5 apr. 1885, pp. 143/II-144/I.

33 Le Ménestrel, 28 apr. 1895, pp. 135/I-136/II.

34 La Rampe illustrée, 20 gen. 1890, p. 25/I-II.

35 Le Guide musical, 12 gen. 1890, pp. 10/II-11/I.

36 Le Ménestrel, 26 lug. 1892, pp. 206/II-208/II.

37 Le Ménestrel, 9 apr. 1893, pp. 119/II-120/II.
} 
on days of low attendance, in 1872 they began to give concerts on Thursday afternoons. [...] In 1893, so performances could be given all year long, they built the Palais d'hiver. This was an immense structure containing a large concert hall and a 50-x-25-meter palm-tree-lined "Palmarium" to which chairs could be brought. The administration also hired Louis Pister, who had worked under Pasdeloup at the Concerts Populaires, and asked him to recruit their musicians from the most prestigious institutions in town - the Société des Concerts du Conservatoire, the Opéra, and the Opéra-Comique. ${ }^{38}$

Molti sono gli articoli di giornale che ci danno informazioni sui concerti diretti da Pister in questa sede, di cui è direttore fino alla fine del 1896: due articoli sono su Le Guide musical, ${ }^{39}$ due su Musikalisches Wochenblatt, ${ }^{40}$ uno su Neue Berliner Musikzeitung, ${ }^{41}$ uno su L'Art musical. ${ }^{42}$ Le altre occorrenze riguardanti questi concerti provengono tutte da Le Ménestrel, che ogni settimana, per tre anni, riporta nel tamburino l'annuncio delle serate al Jardin d'acclimatation: trattandosi di annunci di poche righe, non ritengo utile riportare tutte le occorrenze del settimanale parigino, così come diverrebbe superfluo analizzare il programma di tutti questi concerti; possiamo limitarci alla descrizione offerta da Pasler del parco zoologico, che fornisce molti particolari utili a una panoramica su tali rappresentazioni:

Of the various concert societies in Paris, the Jardin became the most conscious of the diversity of its potential publics [...] The Sunday concerts, performed in the main hall, continued with works by the "best-known masters," or what was considered "easy and likeable music" [...] Besides the price of entry to the zoo (one franc), concert admission cost only twenty centimes to one and a half francs. Four days a week, from 3:00 to 5:00 p.m., the Jardin also initiated concerts for twenty centimes in the Palmarium - Promenade concerts. [...] The Jardin began a new third concert series on Wednesdays with advanced music, which their advance publicity noted would be addressed to a "more initiated" public, those interested in the "progress of art". They hoped the public would enjoy the historical part of their programs and works by young composers. For these, they increased the size of the orchestra from sixty to ninety musicians, occasionally invited guest soloists - pianists, organists, and opera singers - and, to cover the increased expenses, raised ticket prices to from one to four francs. In 1896 they bragged that they had been able to attract the high society of Paris as well as the middle class, business men, and government employees, though not yet much of the working class. ${ }^{43}$

Più nello specifico, per quanto riguarda le scelte artistiche di Pister, si può aggiungere, oltre all'introduzione della musica operistica, la sua volontà di portare al Jardin d'acclimatation più musica francese, da Saint-Saëns, Massenet e Widor, ai meno noti Paul e Lucien Hillemacher e Pierre Letorey. ${ }^{44}$ Il periodo di direzione di Pister al Jardin

\footnotetext{
$\overline{\overline{38} \text { PASLER 2004, p. } 372 .}$

39 Le Guide musical, 30 apr. 1893, pp. 213/I-214/II; Le Guide musical, 7 mag. 1893, pp. 225/II-226/II.

40 Musikalisches Wochenblatt, 21 feb. 1895, pp. 113/I-114/II; Musikalisches Wochenblatt, 25 apr. 1895, p. 240/I-II.

${ }_{41}$ Neue Berliner Musikzeitung, 4 apr. 1895, pp. 137/II-139/I.

42 L'Art musical, 11 gen. 1894, p. 13/r.

43 PASLer 2004, p. 376.

44 Ivi, p. 380.
} 
zoologique d'acclimatation finisce nel 1896, e apprendiamo da Le Ménestrel ${ }^{45}$ che, all'inizio del 1897, gli succede Laffitte.

Prima di vedere Pister impegnato in un incarico duraturo e culturalmente rilevante, bisogna aspettare l'inizio del nuovo secolo; nell'arco di tempo tra il 1896 e il 1901 abbiamo solamente due notizie in merito a dei suoi impieghi come direttore d'orchestra. Il primo ${ }^{46}$ del 1896, è un concerto al Palais du Trocadéro, edificio costruito in occasione dell'Esposizione Universale del 1878, demolito poi nel 1937 per far spazio all'attuale Palais de Chaillot. Il secondo concerto ${ }^{47}$ è invece parte di un festival ospitato al Municipio di Parigi nel 1898.

Saltando al XX secolo, Le Ménestrel ci informa, in due numeri ${ }^{48}$ del fatto che, per decisione del ministro della Pubblica Istruzione e della Direzione delle Belle Arti, Georges Leygues, inizierà una serie di concerti diretti da Louis Pister, dal prezzo accessibile a tutte le tasche, tenuti ogni domenica nel Grand Palais, l'edificio in vetro e muratura costruito negli Champs-Élysées per l'Esposizione Universale del 1900. Di nuovo, eviterò di citare ogni tamburino in cui Le Ménestrel informa di un concerto diretto da Pister: basti sapere che tali concerti, molto popolari, hanno luogo da settembre a novembre del 1901, per venire sospesi a causa del freddo, e continuano fino al 1903. Durante il primo anno di questo ciclo, siamo informati del fatto che il ministro del Commercio, Alexandre Millerand, per evitare la sospensione delle rappresentazioni, concede il permesso a Pister di continuarle in una serra di Cours-la-Reine; ${ }^{49} \mathrm{ci}$ viene anche detto, nello stesso articolo, che il ciclo riprenderà a dicembre del 1901, ma purtroppo non abbiamo altre notizie in merito. Anche fissare nel 1903 la data di conclusione di questo ciclo di concerti non è che un'ipotesi, poiché, dopo il 1902, nessun periodico riporta più informazioni a proposito: il Bollettino municipale di Parigi del 1906, però, attesta una delibera del Consiglio Municipale in merito a due remissioni di debito nei confronti di Pister per l'intervento dei vigili del fuoco a causa di due incendi al Grand Palais avvenuti nel 1902 e nel 1903, fatto che prova come in quell'anno Pister sia ancora direttore dei concerti al padiglione. ${ }^{50}$

Nel 1897, cioè nel periodo tra la direzione del Jardin d'acclimatation e del Grand Palais, Le Ménestrel $^{51}$ e Le Guide musical ${ }^{52}$ pubblicano un annuncio su una serie di concerti mattutini, diretti da Pister, che si terranno presso il Théâtre des FoliesMarigny: il teatro con quel nome (sito all'angolo tra avenue des Champs-Élysées e carré Marigny) viene in realtà demolito nel 1881, e al suo posto sorge il Théâtre Marigny, ma evidentemente la stampa continua a preferire la nomenclatura precedente. Tali

\footnotetext{
$\overline{\overline{45} \text { Le Ménestrel, } 17}$ gen. 1897, pp. 23/II-24/II.

46 Le Guide musical, 21 giu. 1896, pp. 469/I-470/II.

47 Le Ménestrel, 3 apr. 1898, pp. 110/II-112/II.

48 Le Ménestrel, 21 lug. 1901, pp. 231/II-232/II; Le Ménestrel, 25 ago. 1901, pp. 271/II-272/I.

49 Le Ménestrel, 1 dic. 1901, pp. 383/I-384/I.

50 F-Pn 2012-46765, p. 2162/I.

51 Le Ménestrel, 10 ott. 1897, pp. 327/I-328/I.

52 Le Guide musical, 17 ott. 1897, pp. 641/I-642/II.
} 
concerti iniziano nel gennaio del 1903 e continuano almeno fino all'aprile dello stesso anno, quando i periodici smettono di darci notizie a riguardo.

Quella del Théâtre des Folies-Marigny è l'ultima notizia su Pister come direttore d'orchestra; non mancano però le informazioni sul suo impegno come insegnante. Nel 1898, Le Ménestrel ${ }^{53}$ annuncia che Pister, residente in rue d'Alésia 80, aggiunge alle proprie lezioni un corso di accompagnamento e musica d'insieme: è quindi probabile che, già prima di questa data, il compositore si sia dedicato all'insegnamento, sebbene non sia attestato, e che le lezioni siano tenute privatamente a casa del maestro. Poco più avanti possiamo leggere del successo dei suoi studenti a un saggio, così come l'anno successivo è attestato ${ }^{54}$ un successo per un saggio di pianoforte. Probabilmente Pister deve aver insegnato pianoforte fino al 1898, quando aggiunge ai suoi corsi quelli sopra citati. Sappiamo inoltre che, dopo gli ultimi incarichi come direttore, nel 1903 è membro di una giuria per un concorso di strumenti a fiato al conservatorio, ${ }^{55}$ più nello specifico di ottoni. Infine, nel 1908 Pister è citato come professore al Lycée Buffon di Parigi, in un articolo ${ }^{56}$ in cui si rende nota l'accettazione da parte del ministro della Pubblica Istruzione e delle Belle Arti, Gaston Doumergue, dell'ufficio di presidenza dell'Association amicale des professeurs de musique vocale des Lycées et Collèges de l'État (che elegge come presidente lo stesso Pister).

La morte del compositore, ottantaquattrenne, è annunciata su Le Ménestrel ${ }^{57}$ nel 1929; presso le Archives Nationales ne è conservato il certificato di morte, ${ }^{58}$ avvenuta a Parigi il 30 luglio 1929.

Una volta ricostruita la biografia di Pister, sarebbe utile avere qualche notizia sulla sua composizione che più ci interessa, ossia le musiche di scena per La Tosca di Sardou. Purtroppo, i periodici non forniscono nessuna informazione utile in questo senso, a eccezione di una brevissima recensione su Le Figaro: ${ }^{59}$

La Tosca, le nouveau drame de M. Victorien Sardou, dont nous avons constaté l'immense succès, ne contient pas, à proprement parler, de partie musicale; cependant, au premier acte, dans l'église Saint-Andréa, on entend, au loin, des chants religieux; et, au second acte, pendant la réception au palais Farnèse, un orchestre invisible exécute divers airs de danse, parmi lesquels un Menuet et une Saltarelle très réussis. Ces divers morceaux, d'une facture excellente et d'une couleur locale pleine d'originalité, ont été composes par le chef d'orchestre du théâtre, M. Louis Pister, un musicien de talent, qui fut un des collaborateurs les plus assidus de Pasdeloup. Nous devons à l'obligeance de M. Pister de pouvoir offrir à nos lecteurs le Menuet du deuxième acte, réduit pour le piano, et nous le donnons ici, en attendant l'apparition prochaine d'une plaquette qui doit réunir tous les airs et motifs exécutés pendant les deux premiers actes de la Tosca.

\footnotetext{
$\overline{\overline{53} \text { Le Ménestrel, } 30}$ gen. 1898, pp. 38/II-40/II.

54 Le Ménestrel, 15 gen. 1899, pp. 23/I-24/II.

55 Le Ménestrel, 2 ago. 1903, pp. 246/II-248/II; Le Guide musical, 9 ago. 1903, pp. 581/I-584/I.

56 Le Ménestrel, 29 feb. 1908, pp. 70/I-71/II.

57 Le Ménestrel, 23 ago. 1929, p. 376/I-II.

58 F-Pan LH/2172/54.

59 Le Figaro, 14 dic. 1887, p. 2/vI.
} 
A pagina 8 di questo numero di Le Figaro è poi riportato lo spartito della riduzione per pianoforte, nell'edizione di Morris. Probabilmente, quando il critico sostiene che non vi sia una vera e propria parte musicale, intende dire che manca quella componente extra-diegetica che può caratterizzare una partitura per il teatro; in altre parole, non si può evidentemente sostenere che manchi la parte musicale, ma i brani musicali proposti da Pister sono tutti diegetici, ovvero si tratta di musica già pensata come presente all'interno dello svolgimento della trama, fatto di cui abbiamo una prova nelle didascalie di Sardou, le quali citano già i brani musicali poi composti da Pister. Se prendiamo come esempio le composizioni per il secondo atto ci accorgiamo che sono tutte musiche a cui il testo del dramma fa riferimento esplicito: il minuetto, la gavotta e l'adagio di Haydn sono pensati come eseguiti nelle sale di palazzo Farnese, i vari saltarelli sono cantati dalla popolazione romana all'indirizzo della regina Maria Carolina, e la cantata di Paisiello deve essere interpretata da Floria; tutto ciò è quindi all'interno della finzione, non siamo davanti a della musica di commento. Parlando dell'assenza di una musica extra-diegetica, l'articolo involontariamente mette in luce lo stretto rapporto di collaborazione tra il testo verbale della pièce e quello musicale, dato che tutti i brani sono pensati come parte integrante, e perciò imprescindibile, della tragedia.

\section{DESCRIZIONE DELLE FONTI}

Le fonti di cui disponiamo per una ricostruzione delle musiche di scena sono tre: una partitura completa di tutti i numeri musicali e due riduzioni per pianoforte. La prima, AUS-CAnl MUS JWC 340/1, è un testimone manoscritto conservato presso la National Library of Australia. Oltre alla rilegatura, esso si compone di un primo foglio, completamente bianco oltre al titolo, su cui sono stati apposti due timbri: quello più in basso è un grande «1» indicante il numero di catalogo, mentre quello più in alto riporta la dicitura «The property of J. C. Williamson», attore e impresario americano naturalizzato australiano che, dopo aver ottenuto un accordo con Sarah Bernhardt per alcune rappresentazioni in Australia tra cui quelle de $L a T o s c a,{ }^{60}$ deve evidentemente aver acquistato la partitura di Pister giunta nel Paese insieme alla Bernhardt. Dopo questa prima pagina, il manoscritto si compone di 57 fogli pentagrammati: il primo, il terzo e l'ultimo presentano soltanto righi vuoti, il secondo invece attesta l'indicazione «Partition d'orchestre / La Tosca / Drame en cinq actes / de / Victorien Sardou / Musique / de / Louis Pister». Per concludere con questa fonte, il quarto foglio pentagrammato non presenta della musica, ma il testo di un Angelus, pensato probabilmente da Pister per essere recitato o cantillato.

Dando un'occhiata alla partitura delle musiche di La Tosca, ci si accorge che in alcune pagine è presente un timbro rosso, che recita: «Ch[arles] Schnéklüd / Paris, 13, rue des Abbesses / Bureau de copie de Musique». A proposito di questo secondo Carneade, nessun periodico o documento fornisce informazioni utili; l'unica attestazione è in uno studio su Saint-Saëns, in cui si dice che, quando il compositore ha bisogno di far copia-

$\overline{\overline{60} \text { The Argus, } 4 \text { set. }}$ 1890, p. 5/v; The Argus, 9 lug. 1891, p. 5/viI. 
re le parti, le affida a Schnéklüd. ${ }^{61} \mathrm{Si}$ tratta evidentemente di un copista noto a Parigi, su cui però non possiamo aggiungere altro a causa della carenza di fonti. Seppur con qualche difficoltà, è possibile reperire online altri manoscritti editi da questo copista, come un'Ave Maria di Ancel conservata a Madrid, ${ }^{62} \mathrm{o}$ il dramma L'ile désenchantée di Février conservato presso l'Houghton Library dell'Università di Harvard a Cambridge (Mass.). ${ }^{63}$ Quest'ultima biblioteca conserva inoltre un manoscritto, sempre edito da Schnéklüd, delle parti staccate di una pantomima di Viardot-García intitolata Au Japon, interamente consultabile online.$^{64}$ Confrontandole con la partitura di Pister conservata a Canberra, ci si accorge di quanto alcuni elementi siano significativamente simili, come ci si potrebbe aspettare. Le chiavi, le indicazioni dinamiche, i gambi delle crome e in generale l'impaginazione non lasciano dubbi: la partitura "australiana" non può essere considerata un autografo di Pister, ma una copia manoscritta edita da Schnéklüd.

Per quanto concerne le altre fonti, si sono identificate solamente due riduzioni a stampa del minuetto del secondo atto, che evidentemente deve essere stato uno dei brani più apprezzati di questa composizione; entrambe le fonti sono conservate presso la Bibliothèque nationale de France, Département de la musique. La prima, F-Pn VM12-23193, edita da Morris, è quella riproposta sulle colonne di Le Figaro; la seconda, F-Pn VM12-23192, è la medesima riduzione edita da Hartmann. A proposito di quest'ultima fonte, la si può considerare apografa dell'altra, in quanto sono identiche (a eccezione del fatto che F-Pn VM12-23192 non utilizza le stanghette di ripetizione della battuta ma riporta le medesime battute due volte, elemento di per sé trascurabile), ma la seconda aggiunge alcune indicazioni assenti nella prima come anche in AUSCAnl MUS JWC 340/1 (forcelle e indicazioni dinamiche); a margine, bisogna notare come il frontespizio di F-Pn VM12-23193 indichi che si tratta un «Exemplaire offert par l'auteur», fatto che ancor più ci porta a ritenere tale fonte antigrafa dell'altra. Alla luce di ciò, si può considerare F-Pn VM12-23192 un descriptus. A tal proposito, l'edizione critica dei testi di Sardou afferma: «Si le manuscrit autographe de la partition a disparu, il existe deux éditions d'une transcription par le compositeur du menuet du deuxième acte (Hartmann, 1889 e Typ. Morris père et fils, 1889)». ${ }^{65}$

L'indicazione di una fonte rispetto all'altra verrà utilizzata, chiaramente, soltanto nel minuetto, in cui il testimone conservato in Australia sarà comunque ritenuto fonte principale, mentre quelli conservati a Parigi verranno utilizzati per la collazione; in ogni caso, considerandolo un descriptus, si eviterà di tediare il lettore riportando ogni volta l'attestazione di una determinata lezione in F-Pn VM12-23192, ci si limiterà a indicarne l'attestazione in F-Pn VM12-23193.

Davide Ciprandi dciprandi@gmail.com

\footnotetext{
$\overline{61}$ RATNER 2002, p. XVII.

62 E-Mp MUS/MSS/861.

63 US-CAh HTC-LC f (M1500.F43 I4 1925).

${ }^{64}$ US-CAh MS mus 264 (110).

${ }_{65}$ SARdou 2017, p. 246.
} 


\section{RIFERIMENTI BIBLIOGRAFICI}

BARTHes 1988 : R. Barthes, Il brusio della lingua. Saggi critici, IV, a cura di B. Bellotto, Torino, Einaudi, 1988.

Biagi Ravenni - Schickling 2015 : G. Puccini, Epistolario. I (1877-1896), a cura di G. Biagi Ravenni, D. Schickling, Firenze, Leo S. Olschki, 2015.

Biagi Ravenni - Schickling 2018 : G. Puccini, Epistolario. II (1897-1091), a cura di G. Biagi Ravenni, D. Schickling, Firenze, Leo S. Olschki, 2018.

Brooks 2010 : E. Brooks, Sharing the Stage with the Voix d'Or: Sarah Bernhardt and Music in the Belle Époque, tesi di dottorato, Graduate School of Arts and Sciences dell'Università di Washington, 2010.

PAsLer 2004 : J. Pasler, Material Culture and Postmodern Positivism: Rethinking the "Popular" in Late-Nineteenth-Century French Music, in Historical Musicology. Sources, Methods, Interpretations, ed. by S. Crist, R. Montemorra Marvin, Rochester, University of Rochester Press, 2004, pp. 356-387.

Ratner 2002 : S. Ratner, Camille Saint-Saëns (1835-1921). A Thematic Catalogue of His Complete Works, I., The Instrumental Works, Oxford, Oxford University Press, 2002.

SARdou 2012 : V. Sardou, La Tosca, a cura di G. Davico Bonino, Torino, Einaudi, 2012.

Sardou 2017 : V. Sardou, Drames et pièces historiques. II., Fédora, La Tosca, Spiritisme, ed. by I. Moindrot, Parigi, Classique Garnier, 2017.

Schickling 2019 : D. Schickling, Finzioni della realtà. Le "Tosche" di Sardou e di Puccini, in Tosca. Melodramma in tre atti, Milano, Edizioni del Teatro alla Scala, 2019, pp. 69-73.

Viale Ferrero 2003 : M. Viale Ferrero, «Ho l'idea di uno scenario»: i caratteri della scena di Puccini, in La scena di Puccini, a cura di V. Fagone, V. Crespi Morbio, Lucca, Fondazione Ragghianti, 2003, pp. 41-53.

Viale Ferrero 2004 : M. Viale Ferrero, Vedere Tosca, in La Tosca di Puccini e Casa Ricordi, a cura di I. Narici, Milano, Ricordi, 2004, pp. 23-32.

FONTI D'ARCHIVIO

AUS-CAnl MUS JWC 340/1 : L. Pister, La Tosca, partitura manoscritta delle musiche di scena, Parigi, Schnéklüd, 1887.

E-Mp MUS/MSS/861 : L. Ancel, Ave Maria, partitura per voce, violino, violoncello, arpa e organo, Parigi, Schnéklüd, 1906. 
F-Pan LH/2172/54 : documenti sulla vita e sulla carriera militare di L. Pister conservati alle Archives Nationales de France.

F-Pn 2012-46765 : Bulletin municipal officiel de la Ville de Paris, 1906.

F-Pn 4-VM-535 : Le Conservatoire national de musique et de déclamation: documents historiques et administratifs, Parigi, Imprimerie Nationale, 1900.

F-Pn VM12-23192 : L. Pister, Menuet de La Tosca, riduzione per pianoforte, Parigi, Georges Hartmann et Compagnie éditeurs, 1889.

F-Pn VM12-23193 : L. Pister, La Tosca. Menuet (2 ${ }^{\mathrm{me}}$ Acte), riduzione per pianoforte, Parigi, Typographie Morris père et fils, 1889.

US-CAh HTC-LC f(M1500.F43 I4 1925) : H. Févier, L'ile désenchantée, partitura manoscritta, Parigi, Schnéklüd, 1925.

US-CAh MS mus 264 (110) : P. Viardot-García, Au Japon, parti staccate manoscritte, Parigi, Schnéklüd, data sconosciuta.

\section{Periodici}

Asmodeo (settimanale milanese) : 1889 (20 mar.).

Corriere della Sera (quotidiano milanese) : 1889 (13 feb.); 1895 (8 ott.).

La France musicale (settimanale parigino) : 1869 (18 lug.).

La Rampe illustrée (settimanale parigino) : 1890 (20 gen.).

L'Art musical (settimanale parigino) : 1894 (11 gen.).

Le Figaro (quotidiano parigino) : 1887 (25 nov.;14 dic.); 1889 (4 dic.).

Le Guide musicale (settimanale brussellese) : 1890 (12 gen.); 1893 (30 apr.; 7 mag.); 1896 (21 giu.); 1897 (17 ott.); 1903 (9 ago.).

Le Ménestrel (settimanale parigino) : 1869 (8 ago.); 1884 (7, 14, 21 dic.); 1885 (5 apr.); 1886 (9 mag.); 1887 (25 set.); 1890 (5 gen); 1892 (26 giu.); 1893 (9 apr.); 1894 (23 set.; 28 ott.; 4, 25 nov.; 16, 23, 30 dic.); 1895 (6, 13, 20, 27 gen.; 3, 10 feb.; 3, 10, 17, 24 mar.; 7, 14, 21, 28 apr.; 5 mar.; 12, 19 mag.; 9 giu.; 13, 20, 27 ott.; 3, 10, 17, 24 nov.; 1, 8, 15, 22, 29 dic.); 1896 (6, 12, 19, 26 gen.; 2, 9, 16, 23 feb.; 1, 8, 12, 15, 29 mar.; 5, 12, 19, 26 apr.; 10, 24, 31 mag.); 1897 (17, 24 gen.; 10 ott.); 1898 (30 gen.; 3 apr.); 1899 (15 gen.); 1901 (21 lug.; 25 ago.; 1, 8 set.; 20 ott.; 3, 10 nov.; 1 dic.); 1902 (24 ago.); 1903 (4, 11, 18, 25 gen.; 1, 8 15, 22 feb.; 1, 8, 15, 29 mar.; 5 apr.; 2 ago.); 1907 (12 gen.); 1908 (29 feb.); 1929 (23 ago.).

Le Petit Parisien (quotidiano parigino) : 1887 (26 nov.).

Le Temps (quotidiano parigino) : 1884 (16 dic.); 1887 (28 nov.).

Le XIX $X^{\mathrm{e}}$ siècle (quotidiano parigino) : 1887 (26 nov.). 
L'Indipendente (quotidiano triestino) : 1889 (30 gen.).

Musikalisches Wochenblatt (settimanale berlinese) : 1885 (1 gen.; 21 feb.; 25 apr.).

Neue Berliner Musikzeitung (settimanale berlinese) : 1895 (4 apr.).

The Argus (quotidiano melbourniano) : 1890 (4 set.); 1891 (9 lug.).

The Musical World (settimanale londinese) : 1889 (13, 27 lug.; 7 dic.). 
Partition d'orchestre

\title{
La Tosca
}

\section{Drame en cinq actes \\ de \\ Victorien Sardou}

\author{
Musique \\ de \\ Louis Pister
}




\section{$1^{\text {er }}$ acte}

\section{$\mathrm{N}^{\circ} 1$ \\ [Angelus]}

Récitation (réplique): [Mario: Allez, allez... Voici le sacristain,] et vite le danger nous talonne[!]

Récit. (Basse solo): Angelus Domini annunciavit Mariae,

Chœur (Ténors, Soprani): et concepit de Spiritu Sancto.

Récit.: $\theta$ Ave Maria gratia plena, Dominus tecum, benedicta tu in mulieribus, et benedictus fructus ventris tui, Jesus.

Chœur: \& Sancta Maria, Mater Dei, ora pro nobis peccatoribus, nunc et in hora mortis nostrae. Amen.

Récit.: Ecce ancilla Domini.

Chœur: Fiat mihi secundum verbum tuum.

Récit.: Ave Maria, etc. etc. au signe $\theta$

Chœur: Sancta Maria, etc. etc. au signe $\$$

Récit.: Et verbum caro factum est,

Chœur: et habitabit in nobis.

Récit.: Ave Maria, etc. etc. au signe $\theta$

Chœur: Sancta Maria, etc. etc. au signe $\$$

Récit.: Ora pro nobis Sancta Dei genetrix,

Chœur: ut digni efficiamur promissionibus Christi.

Finir la récitation à la réplique: [Eusèbe, allant allumer les cierges devant la Madone: Alors, Votre Excellence a] déjà entendu parler de cette victoire de Marengo[?] 
Réplique:

[Eusèbe: Oui... oui... le Bonaparte en carton..

$\mathrm{Ah}$ ! Ah! Celui qui franchit les Alpes avec ses

canons!...] Farceur[,] va[!] C'est à se

$\mathrm{N}^{\circ} 2$

tordre[!....

Psaume à 4 voix

er acte
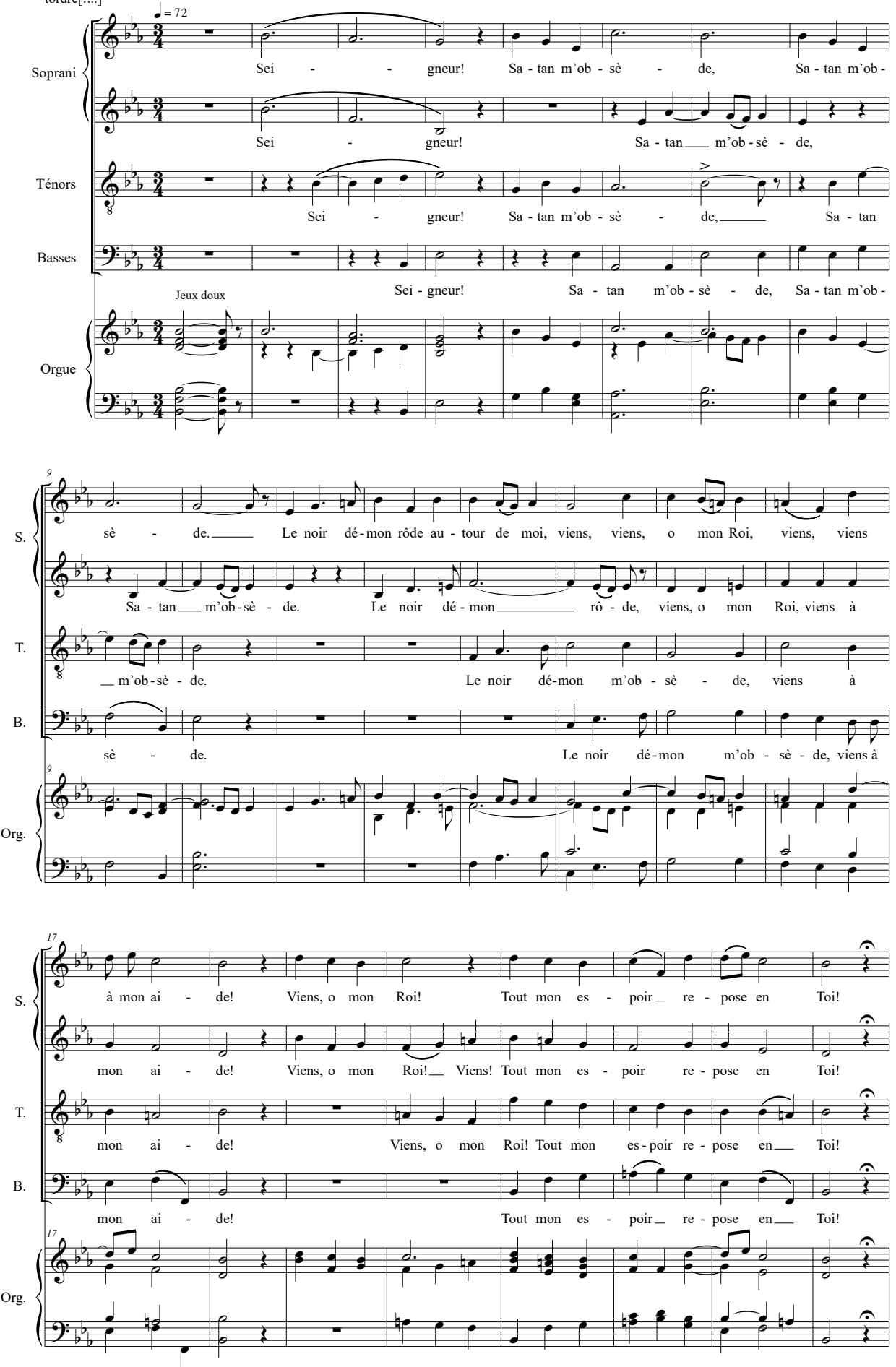

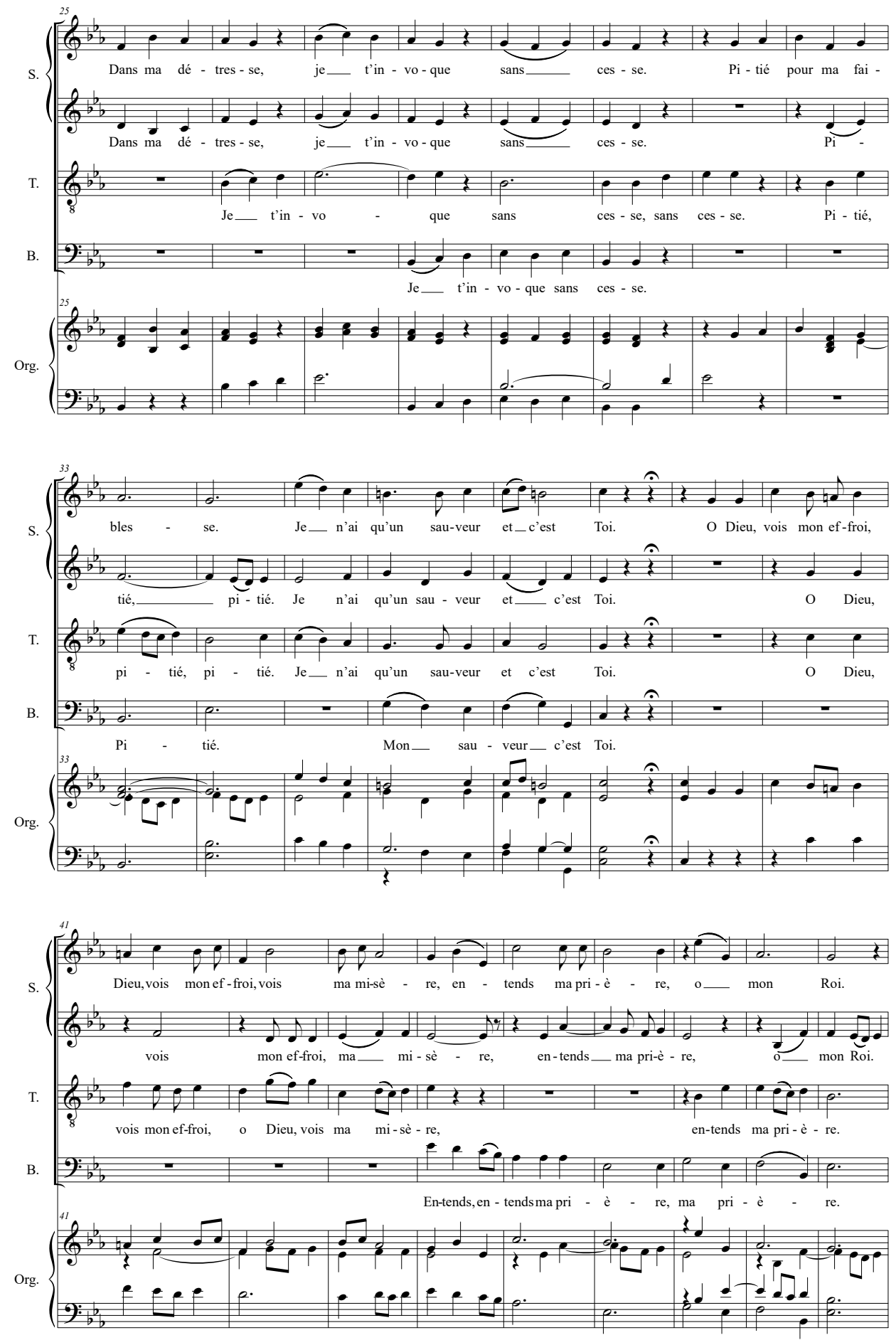


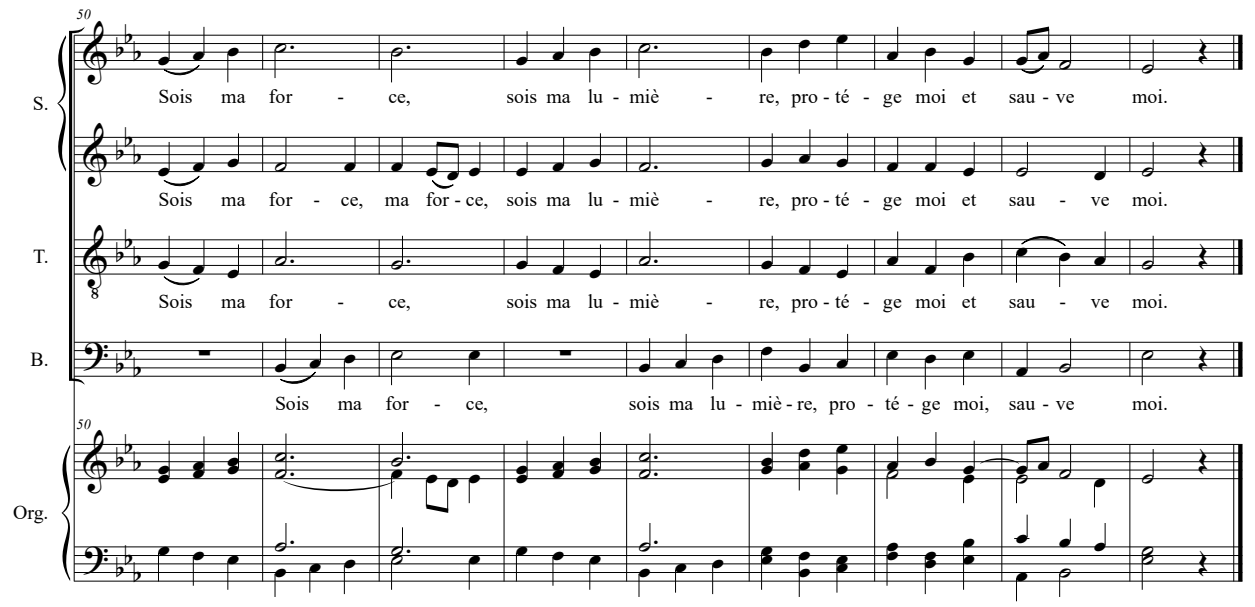




\section{Réplique:}

[Scarpia: Aucune personne suspecte?

L'agent: Aucune.

Scarpia:] Nous l'avons manqué de

quelques minutes[!]

$N^{\circ} 3$

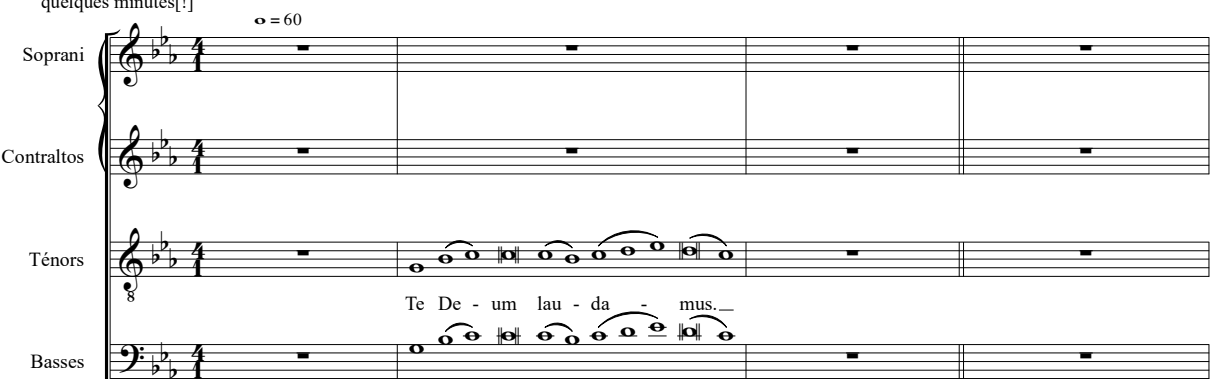

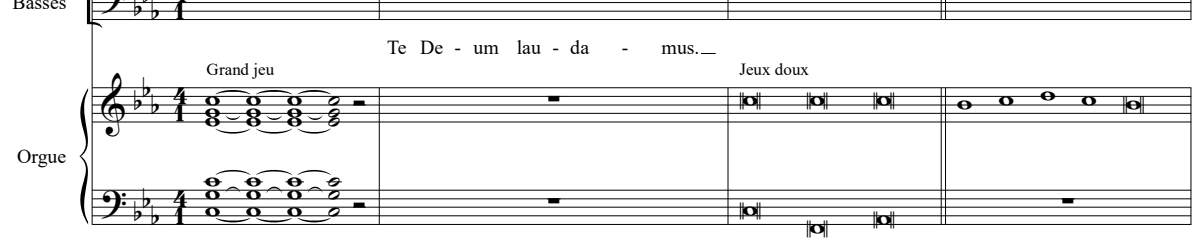

Suivez la scène: prendre après la double barre et dans le mouvement indiqué à la réplique: [Scarpia:] Et prions la Sainte Madone [... (Il se courbe devant elle.) de bénir nos efforts dans cette autre guerre que nous faisons à l' impiétét....]

T.
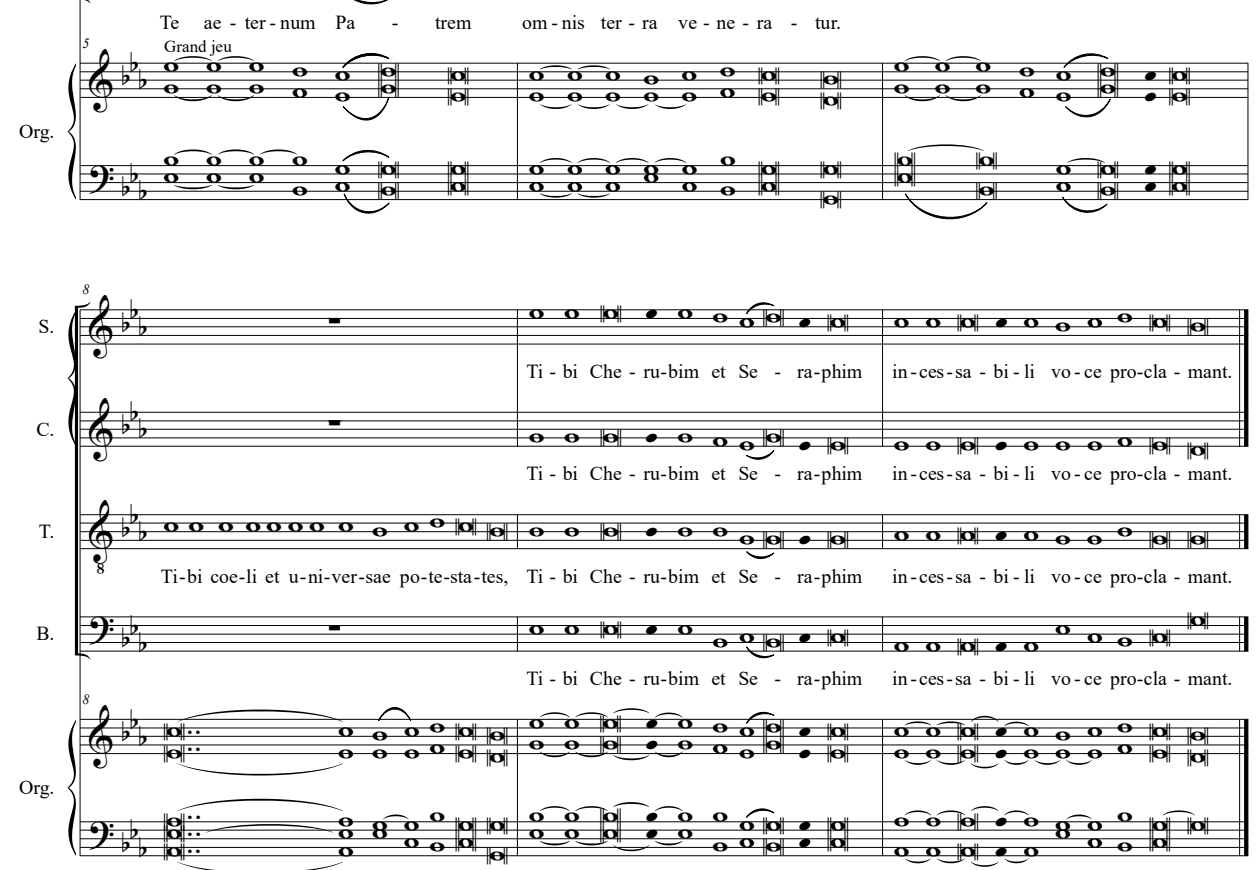


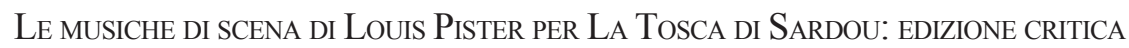

Menuet
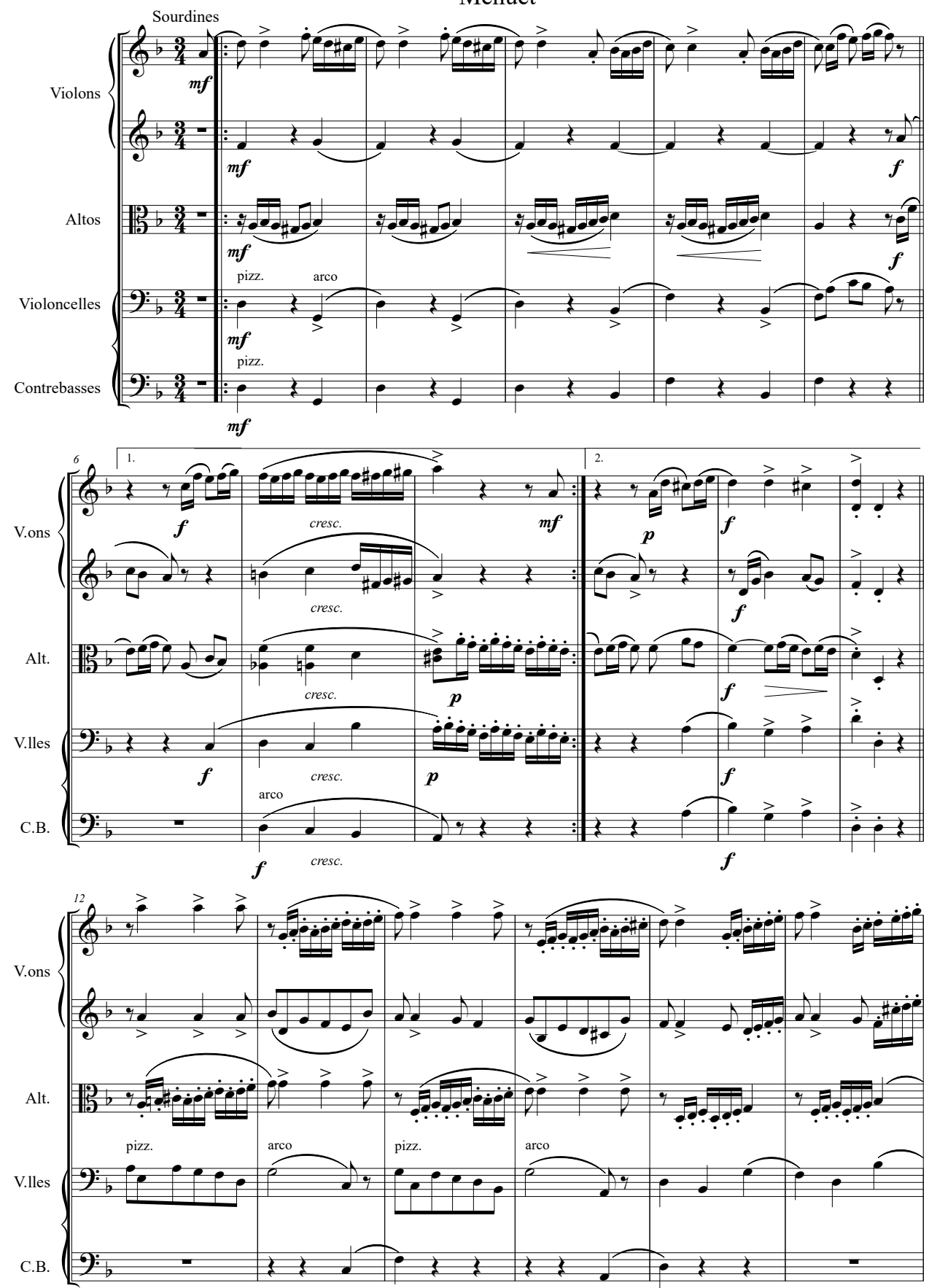

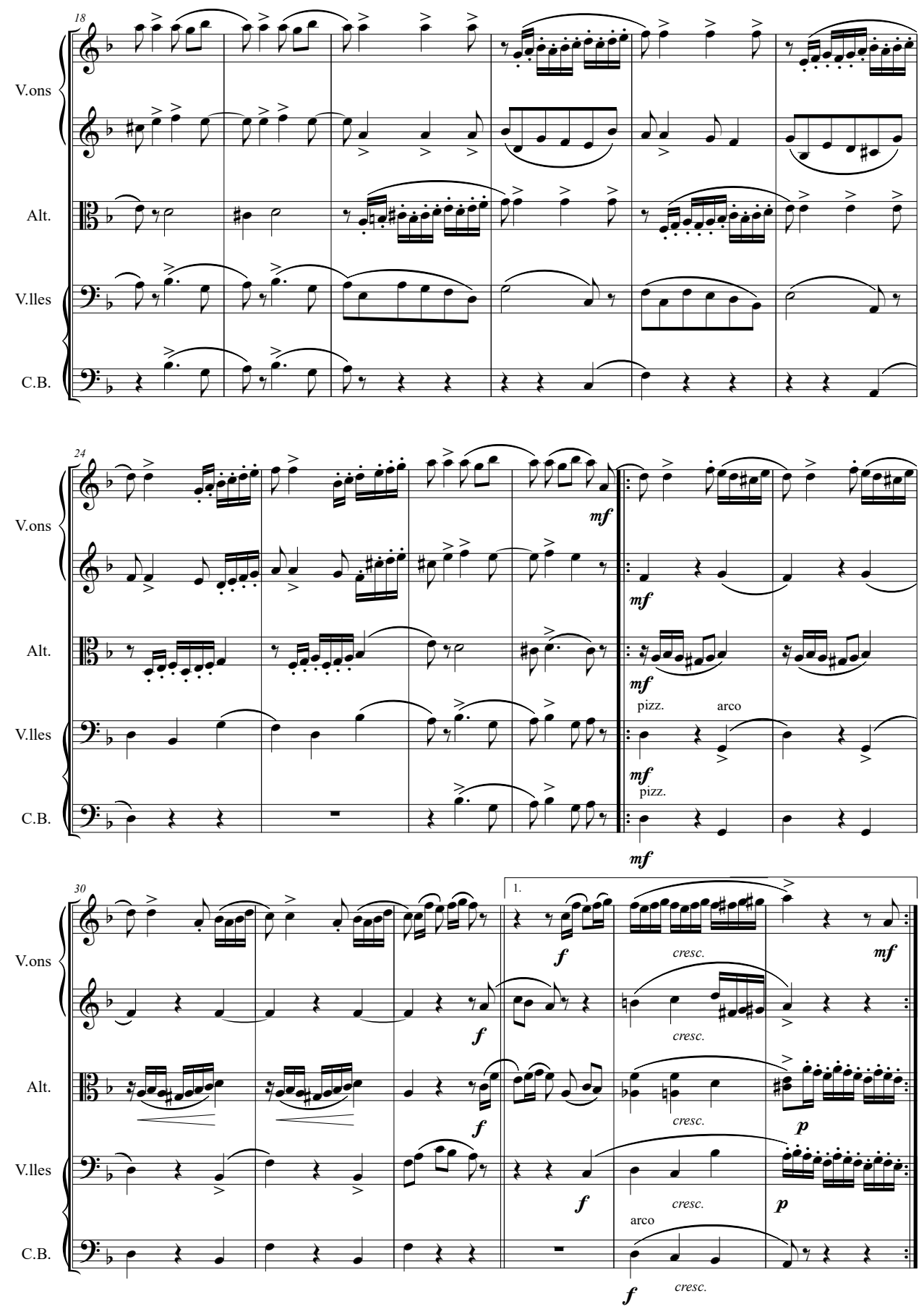


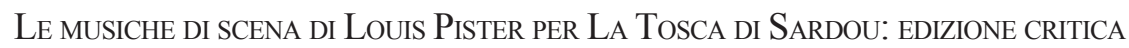
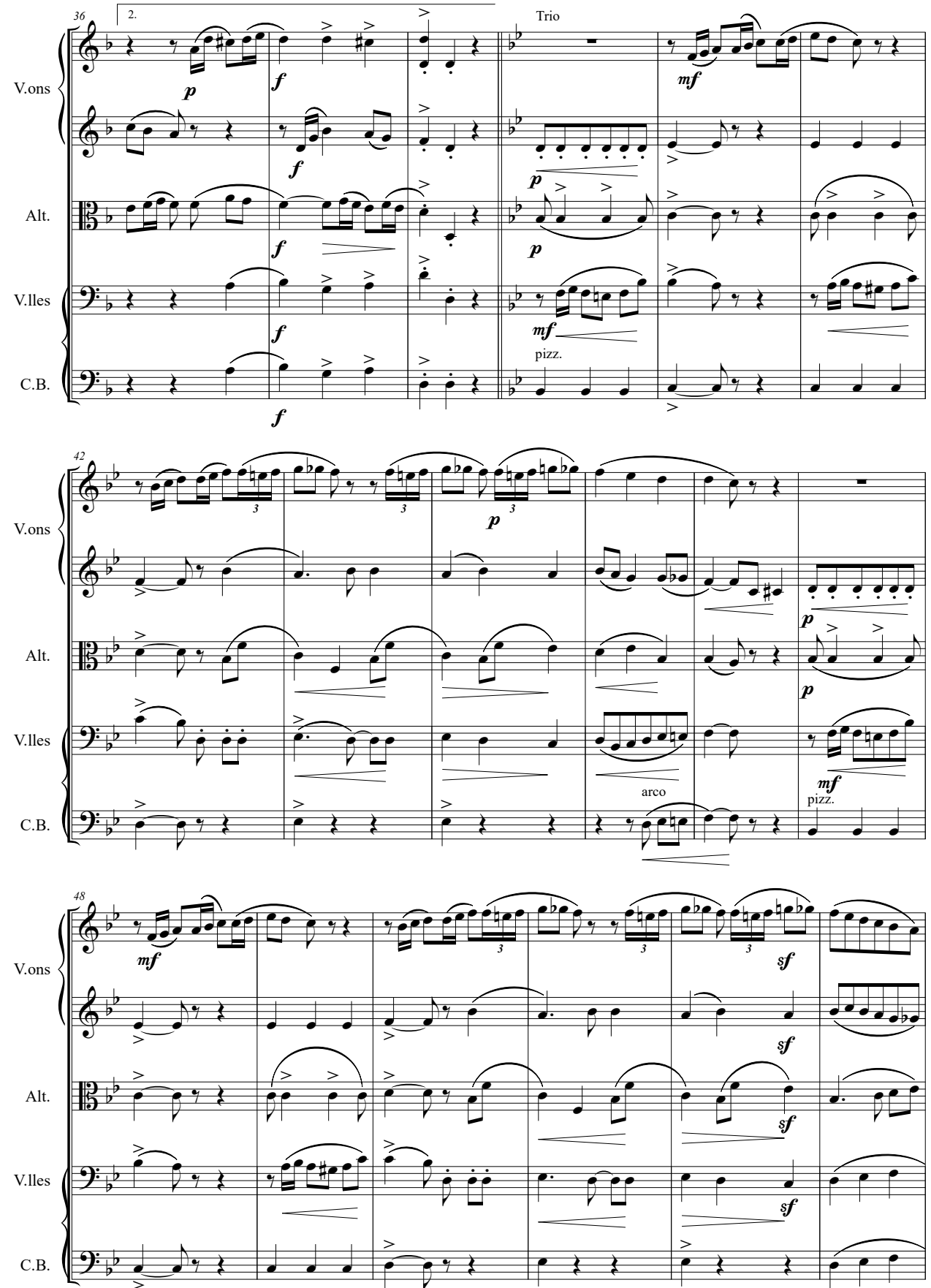

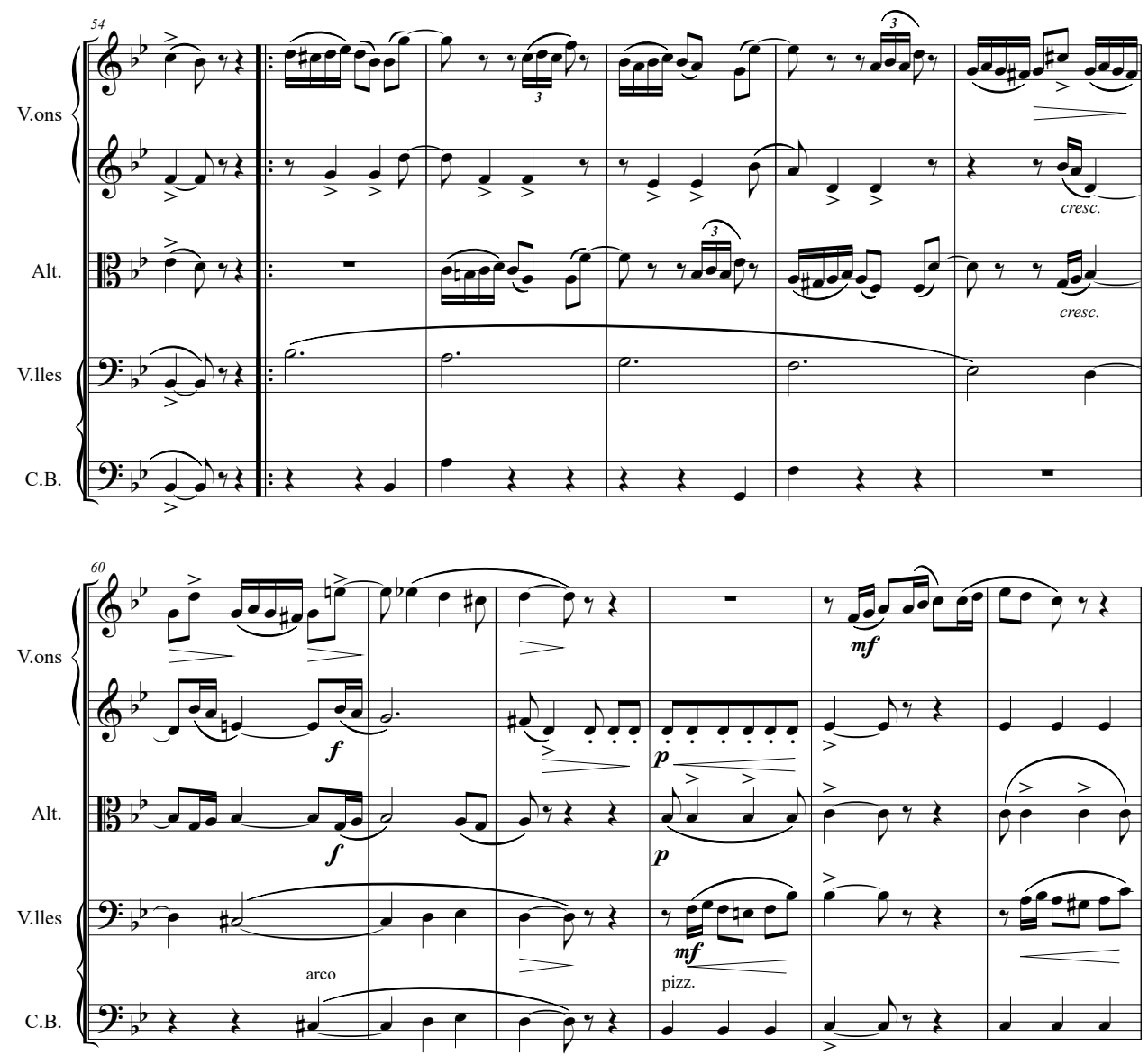

$$
=
$$

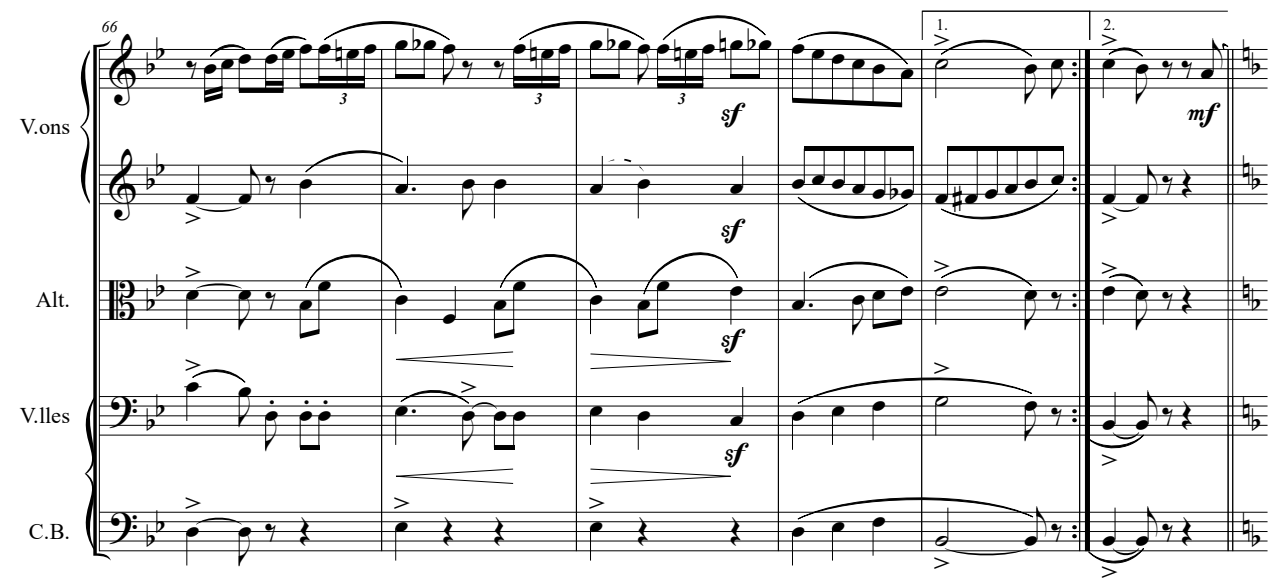




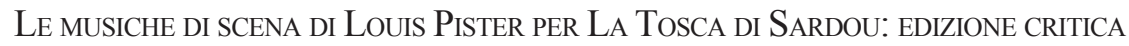
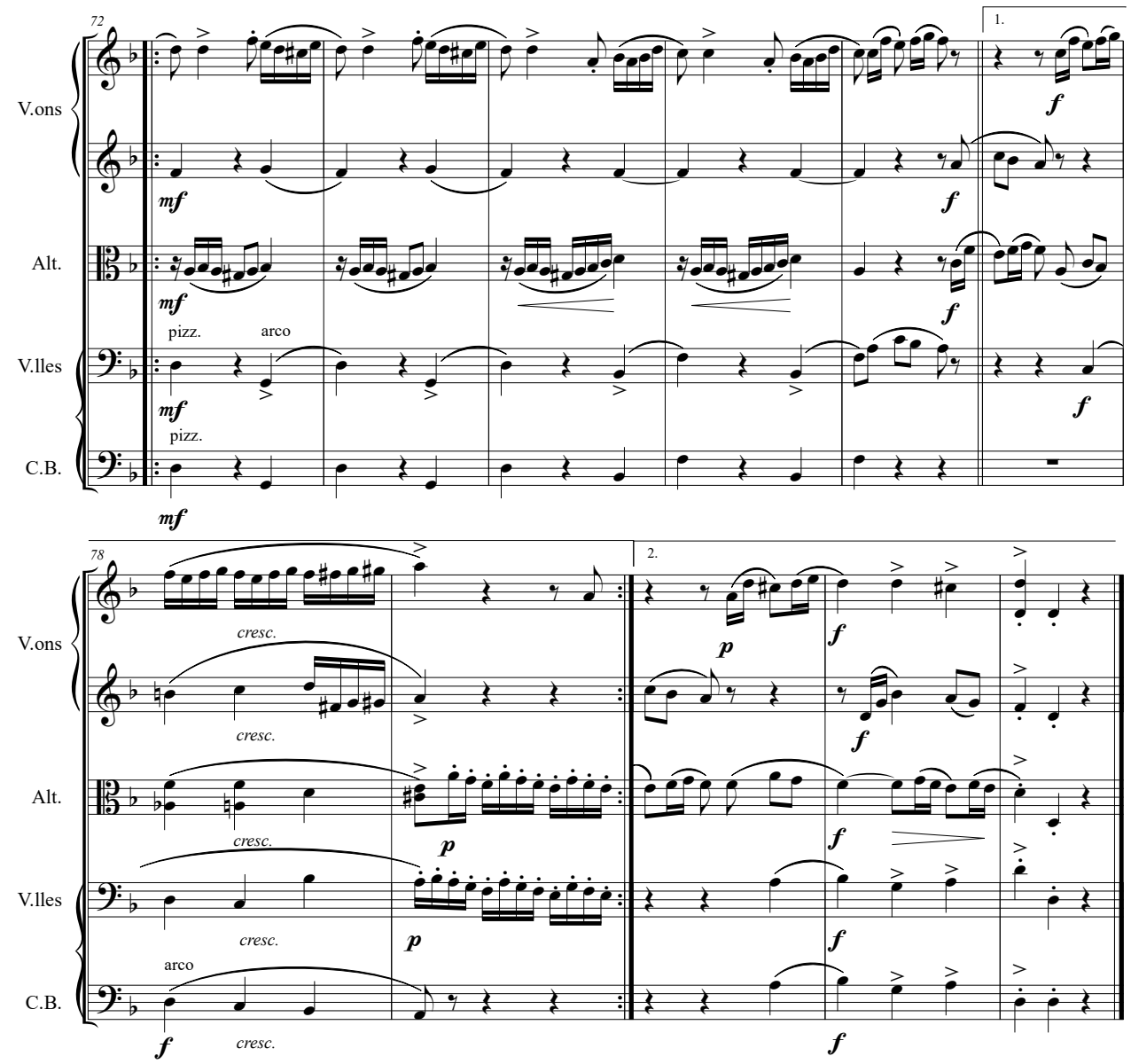

Finir le menuet à la replique:

[La Princesse: Oh! pardon, cela diffère. [(à Attavanti qui descend à eux.)] N'est ce pas[,] marquis[?] 

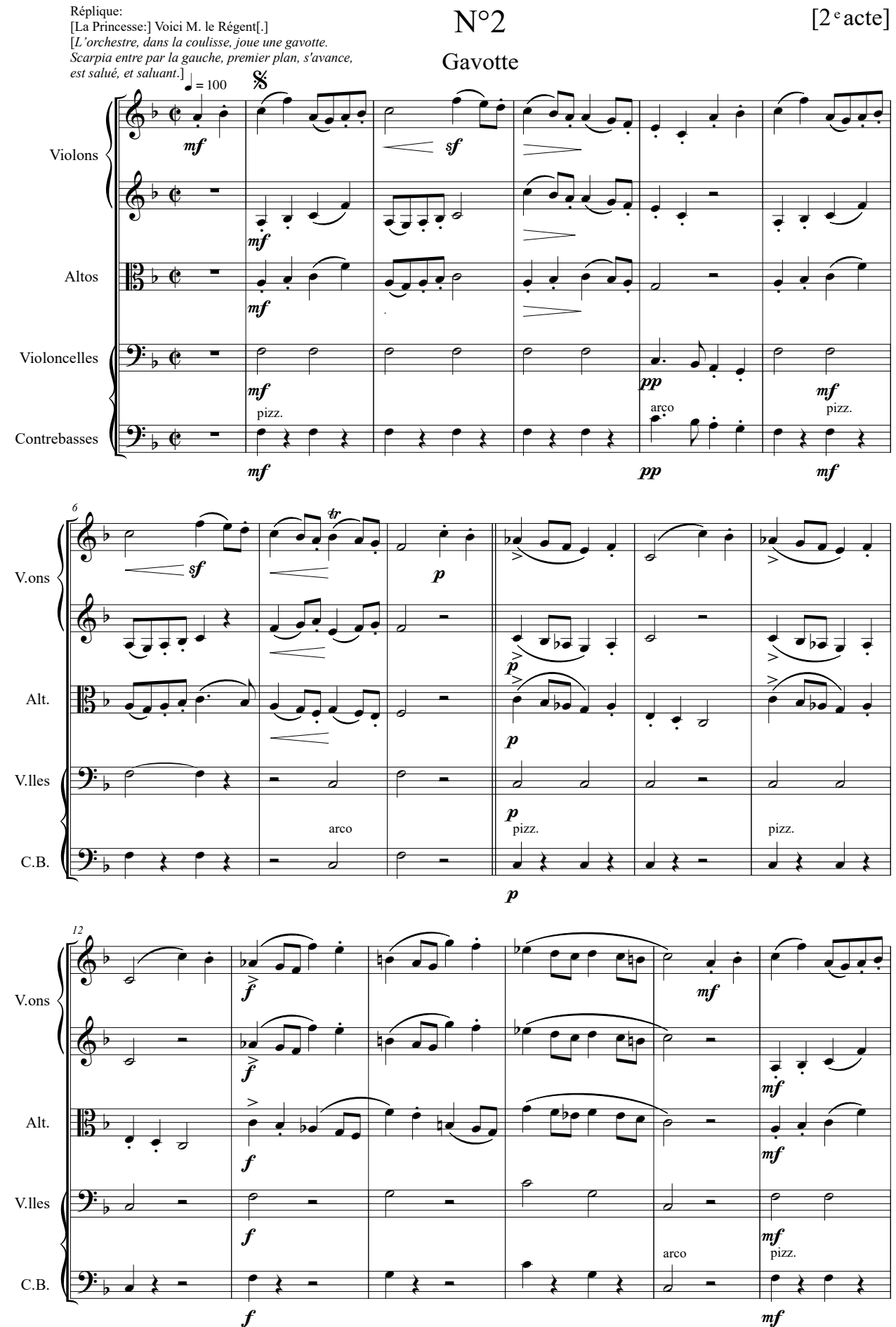
Le MUSICHE DI SCENA di LOUIS PISTER PER LA TOSCA DI SARDOU: EDIZIONE CRITICA

163
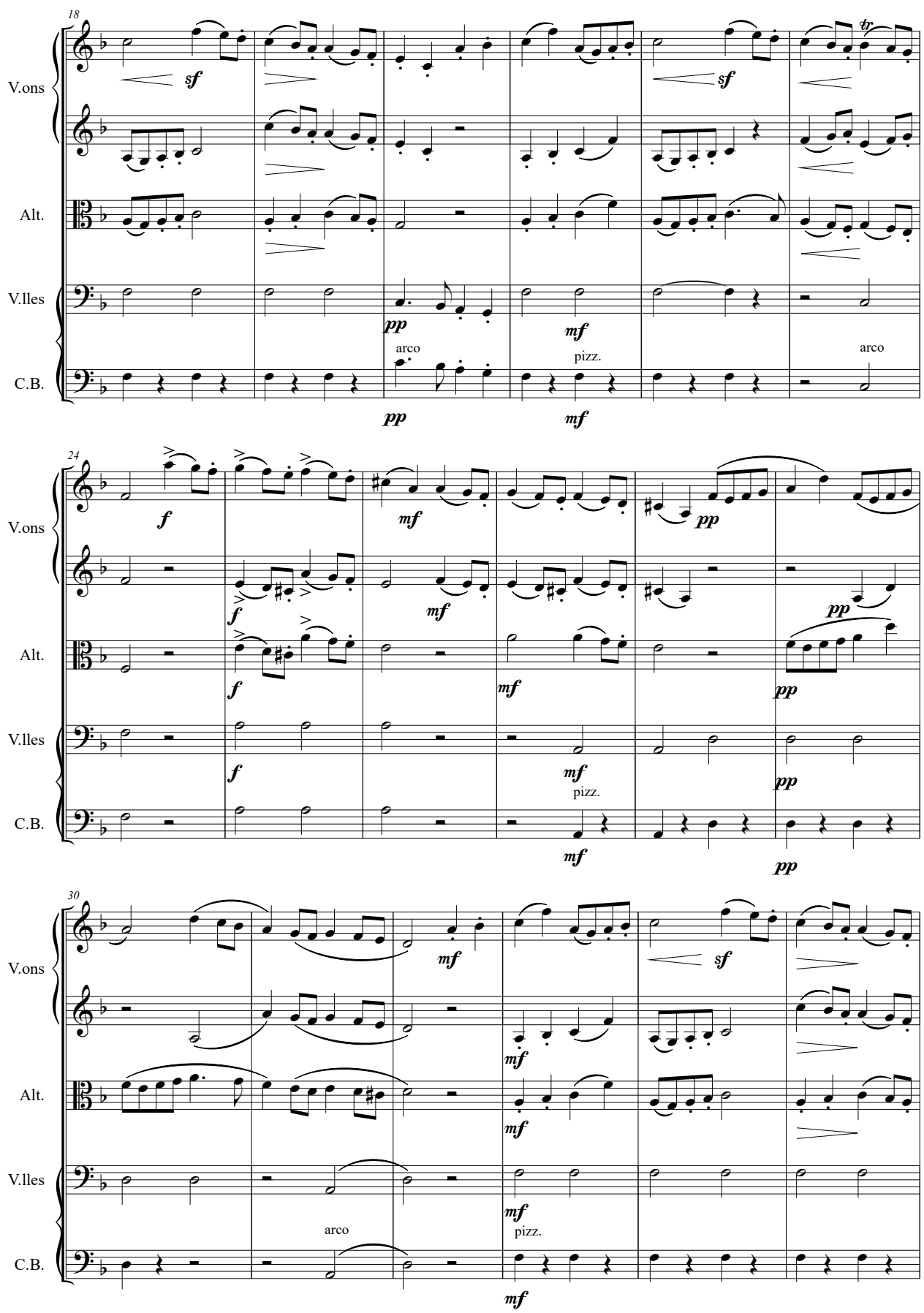

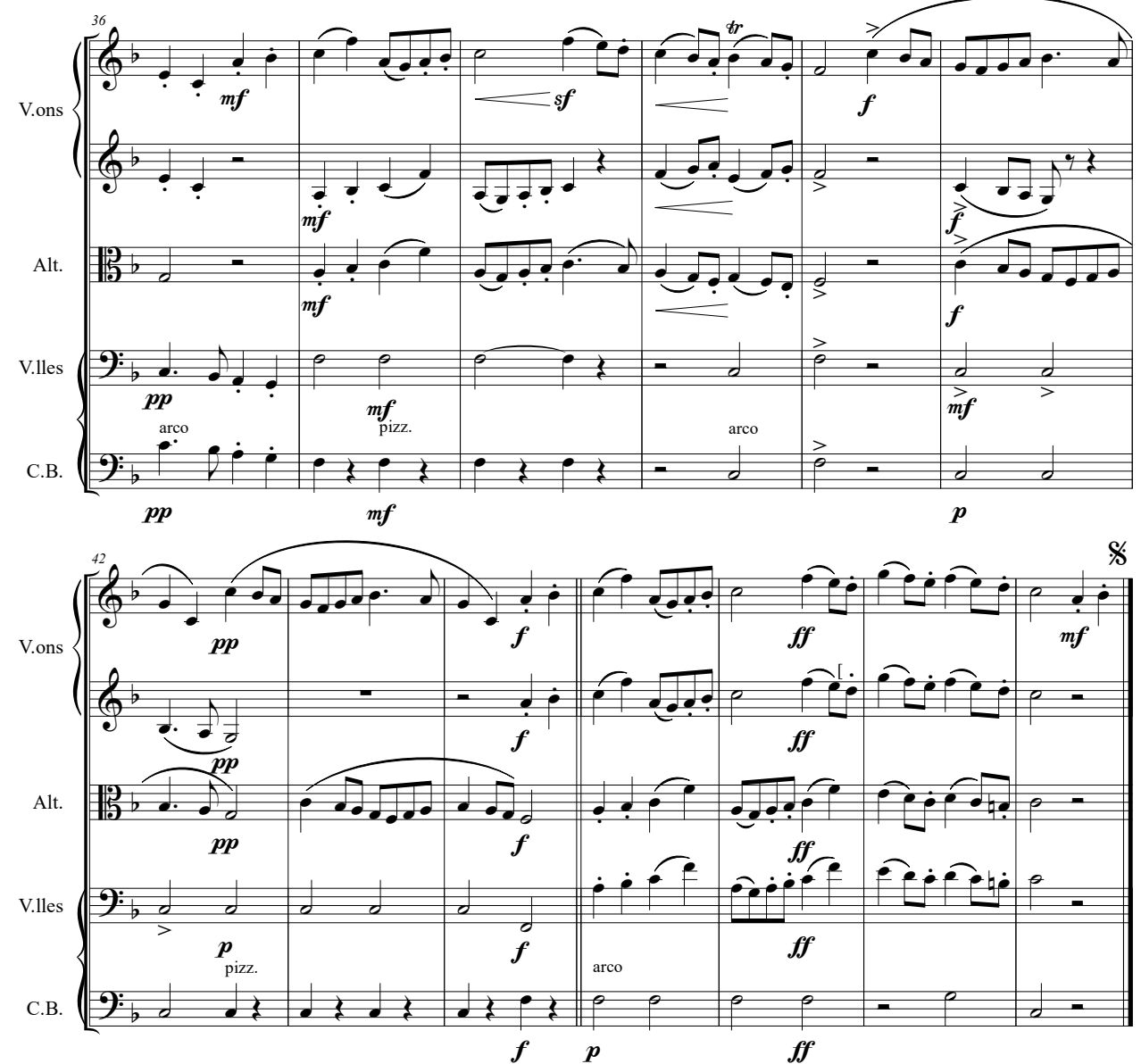

Finir à la réplique:

[Schiarrone, debout: Oui, Excellence.

Scarpia:] Qu'ils y restent[!...] Et toujours à ma portée.

[La musique cesse. Schiarrone sort par la gauche.] 


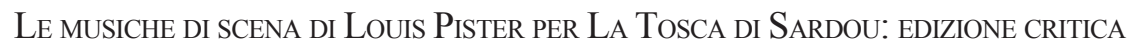

Réplique:

[Marie-Caroline: Tant mieux

$\mathrm{N}^{\circ} 3$

[2 ${ }^{\mathrm{e}}$ acte]

puor toi, car j'aurais bien du ma

à] conjurer la mauvaise humeur

Saltarelle Chœur et Quatuor

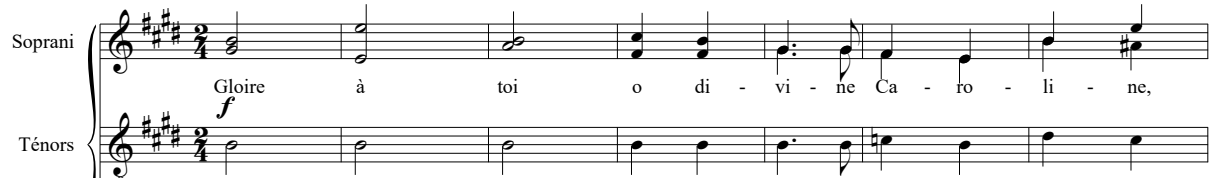

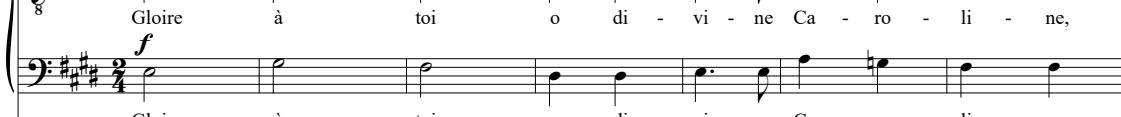

Tambour

de Basque
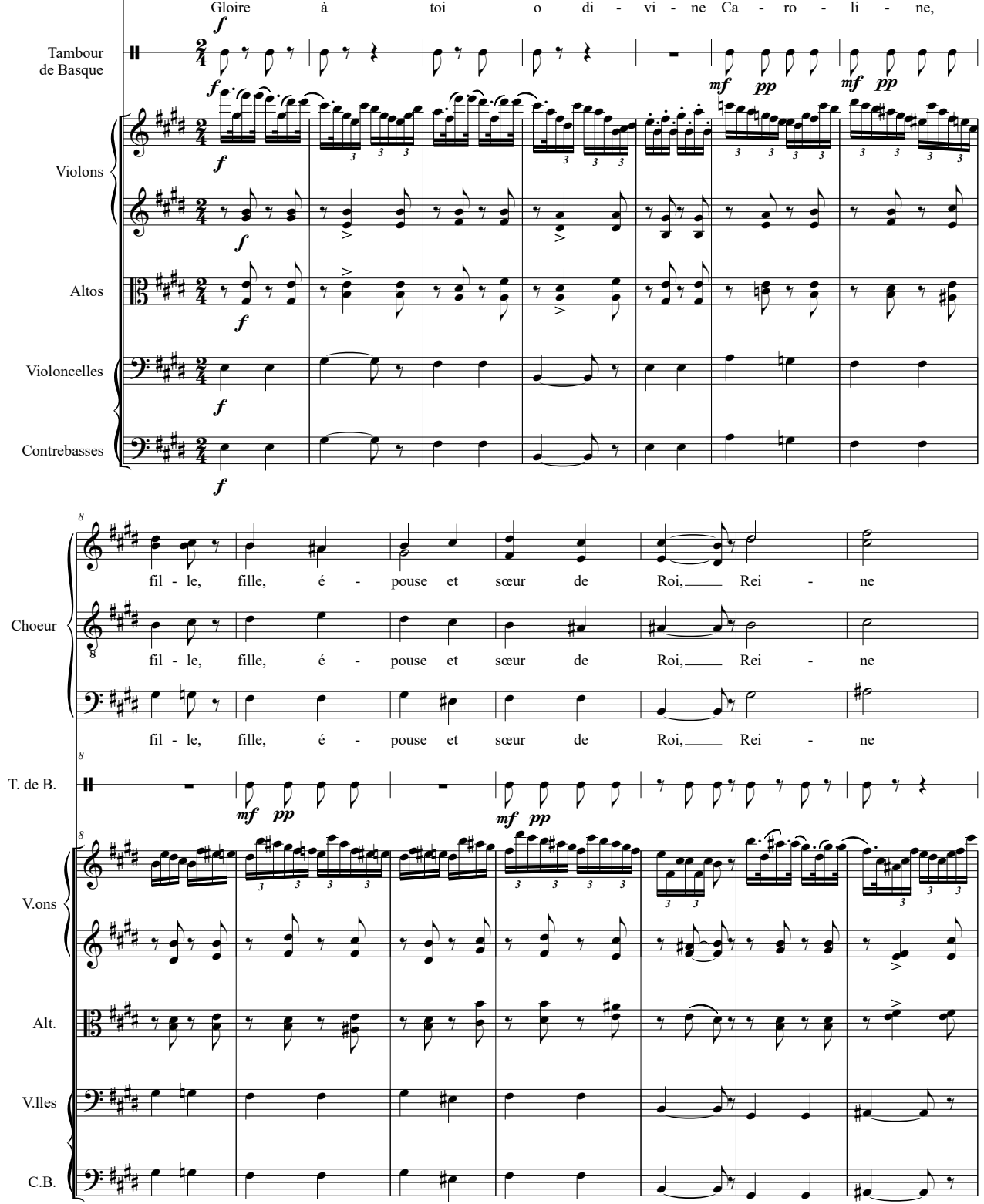

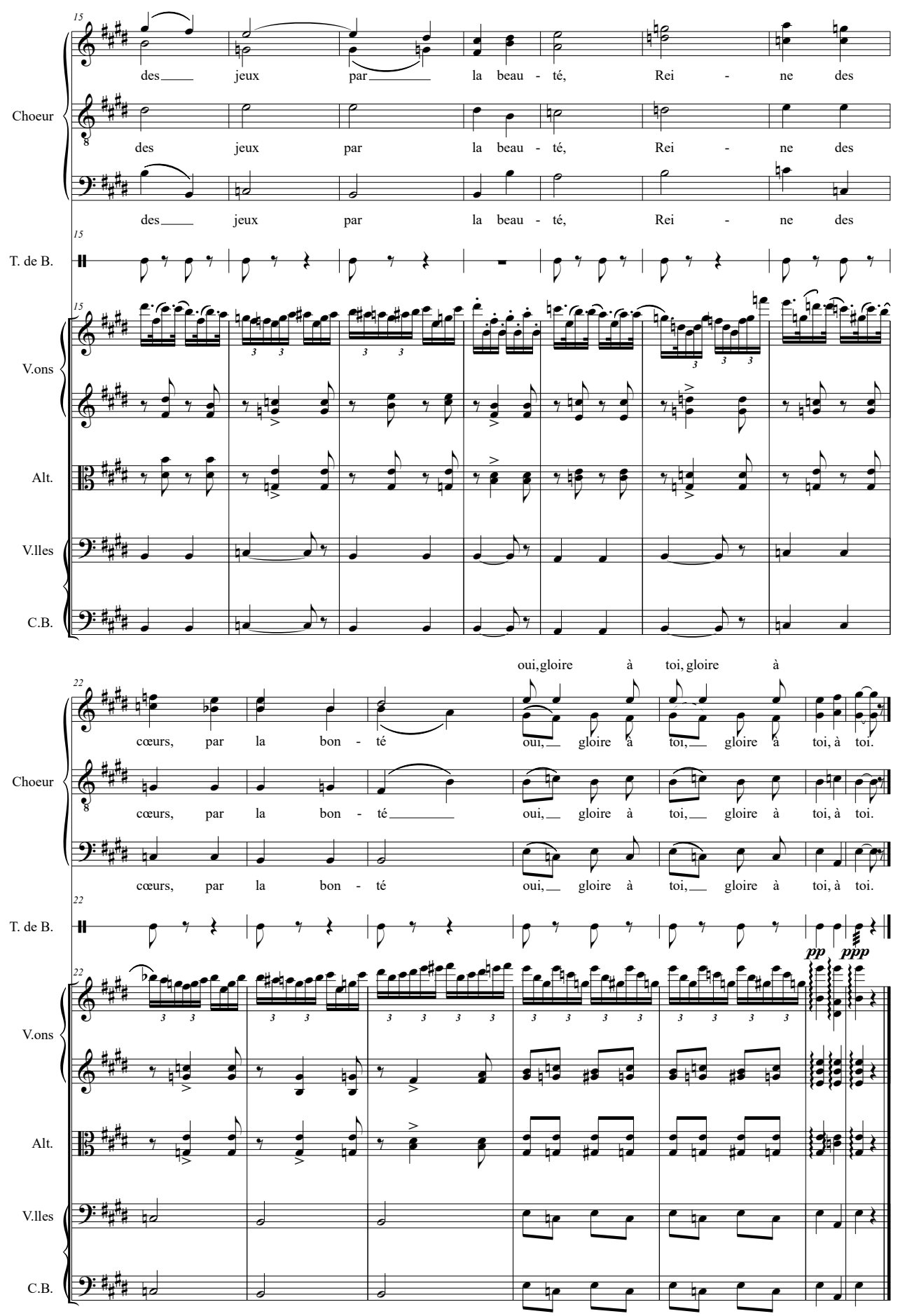


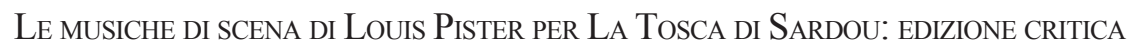
Réplique:
[Scarpia: Et] à la bonne heure, je me retrouve[!]
$\mathrm{N}^{\circ} 4$
[2 ${ }^{\mathrm{e}}$ acte]

$[\ldots .].[$ ['orchestre, dans les salons, joue l'andante de

la symphonie de Haydn en ré majeur.]

Andante
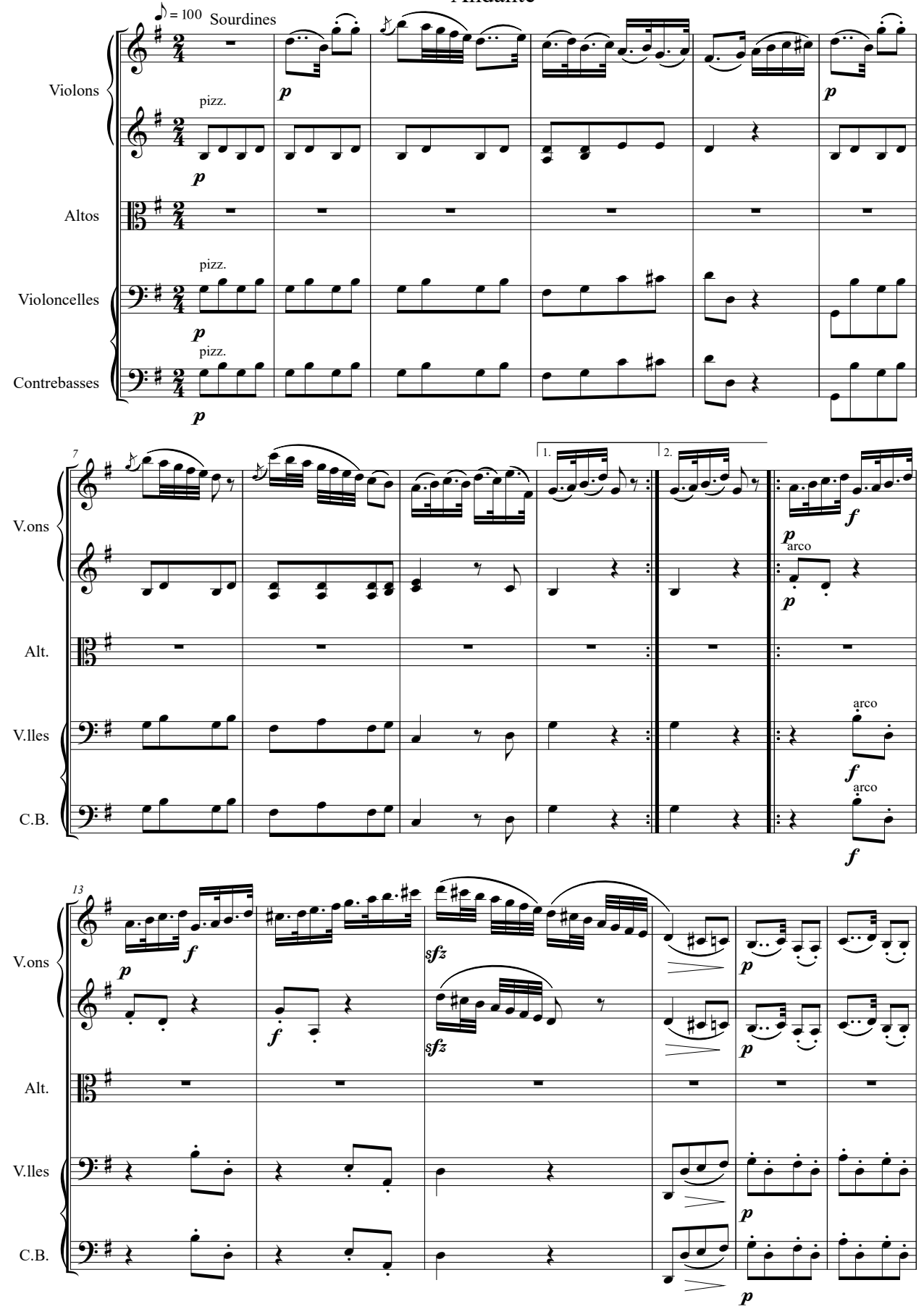

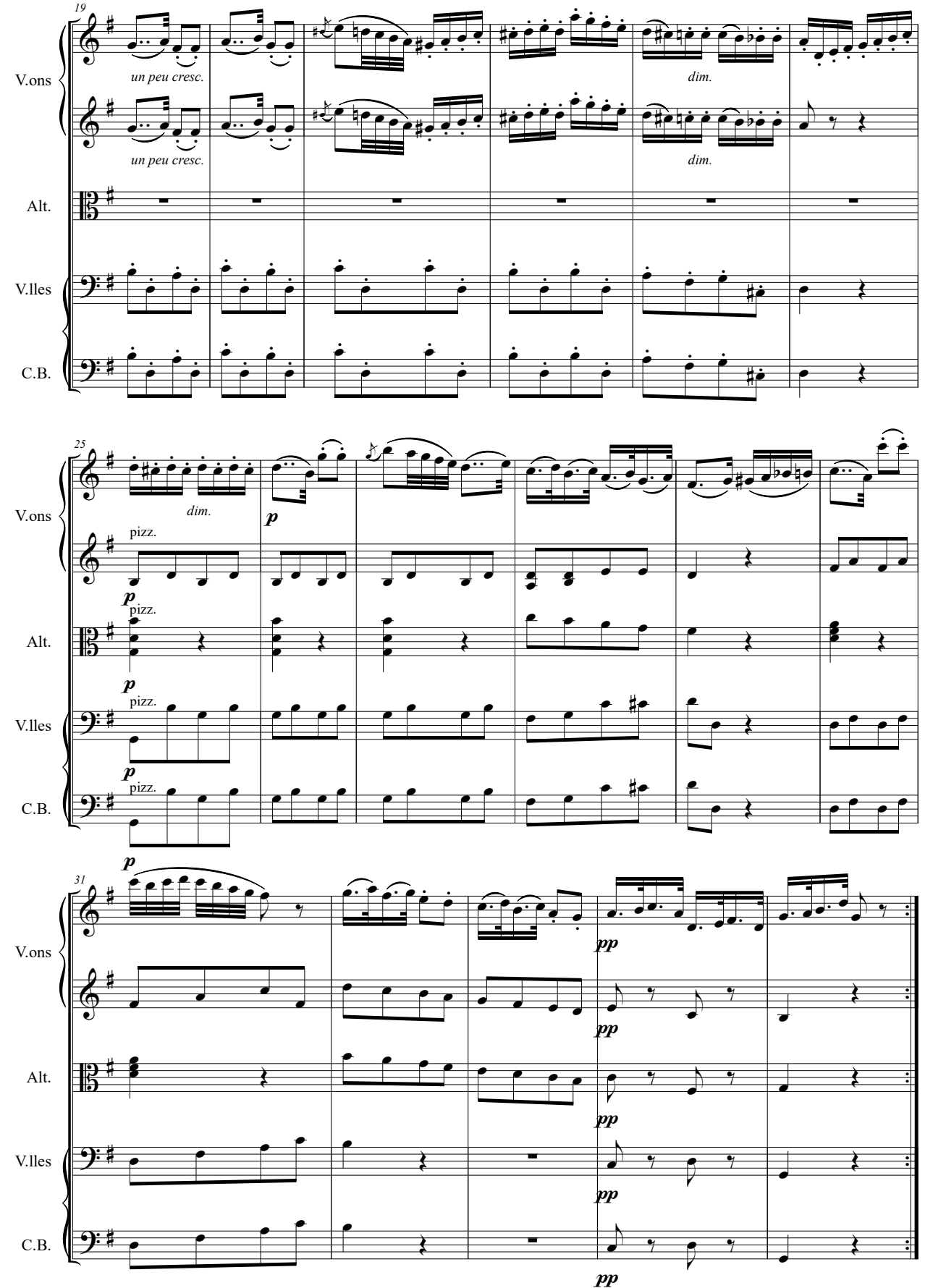
Le MUSiche di scena di Louis Pister Per La Tosca di SARdou: edizione critica

Réplique:

[Paisiello, retournant à ses

musiciens:] Bémol[!] Bémol[!

$\mathrm{N}^{\circ} 5$

[2 e acte]

[Les chants et les fanfares Saltarelle/Fanfare Chœur et Quatuor
reprennent sur la place.]

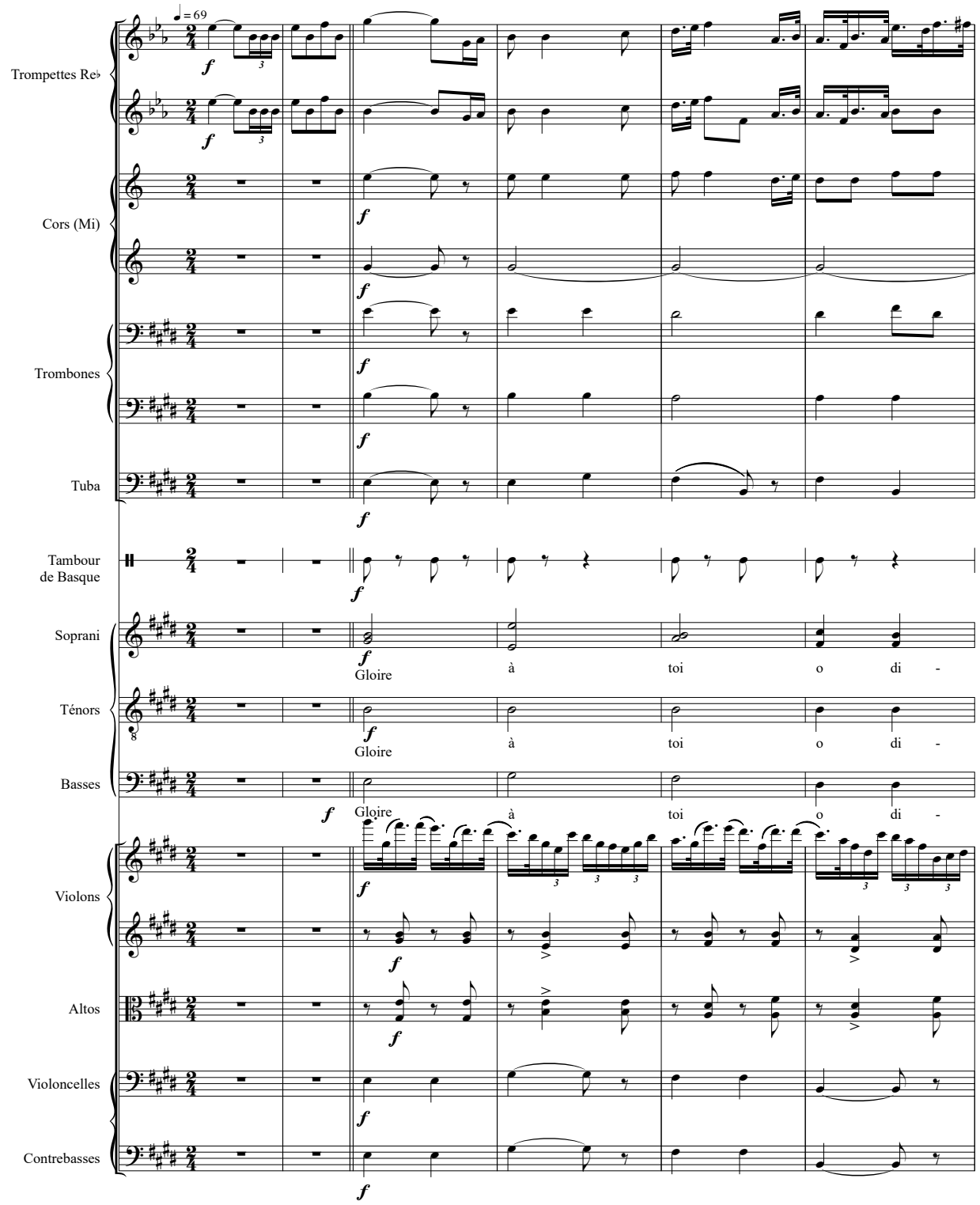




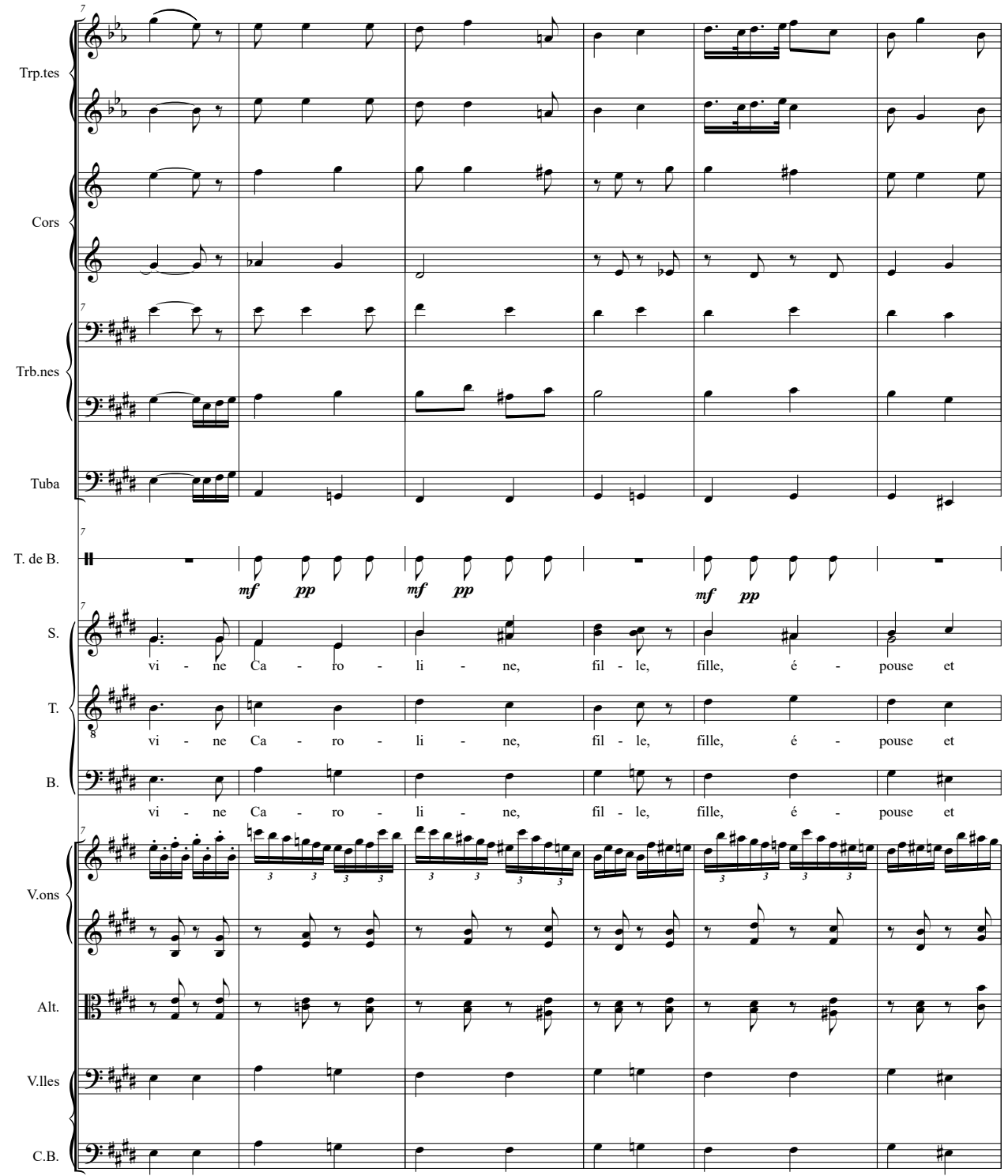




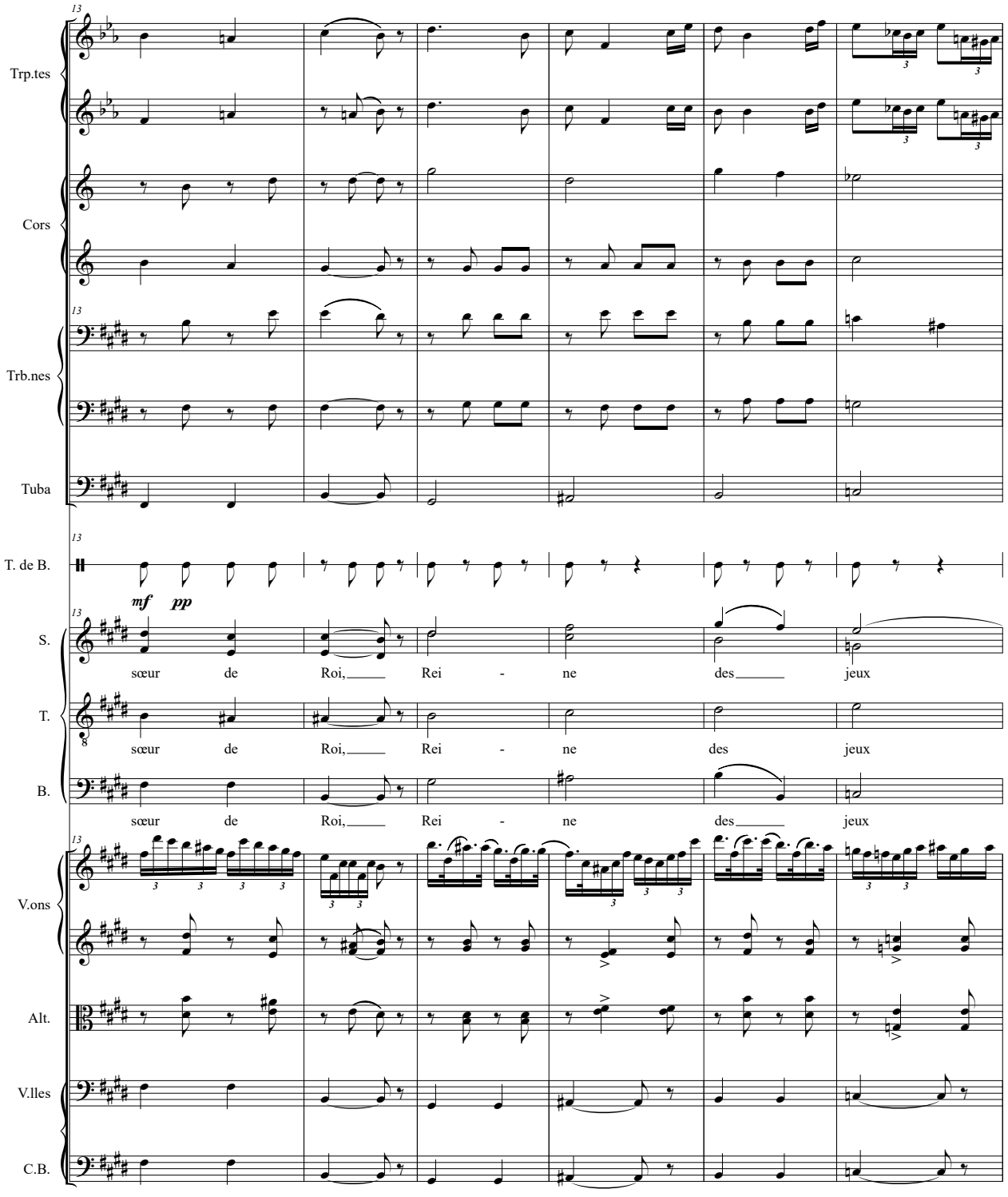



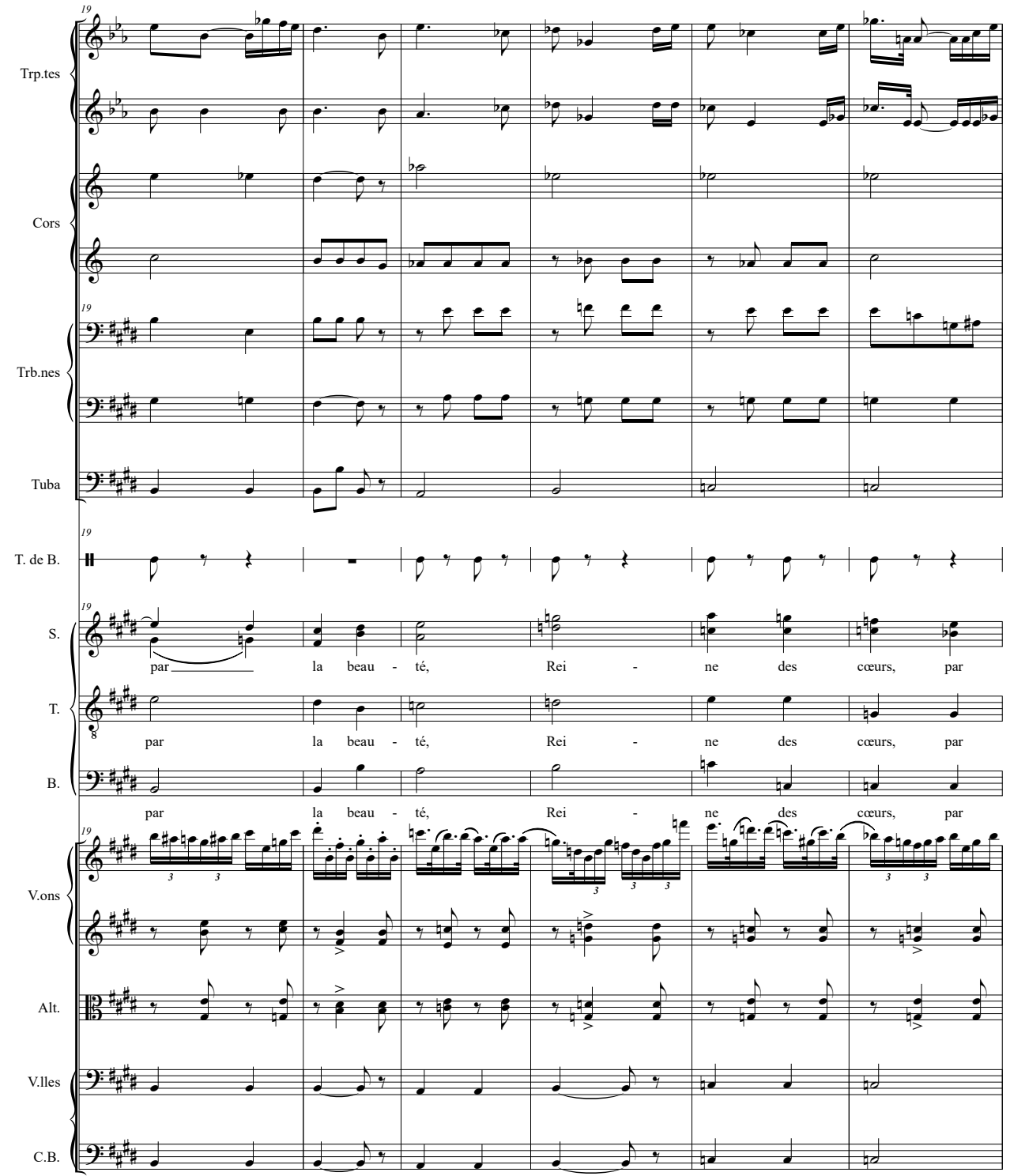


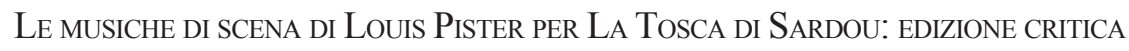

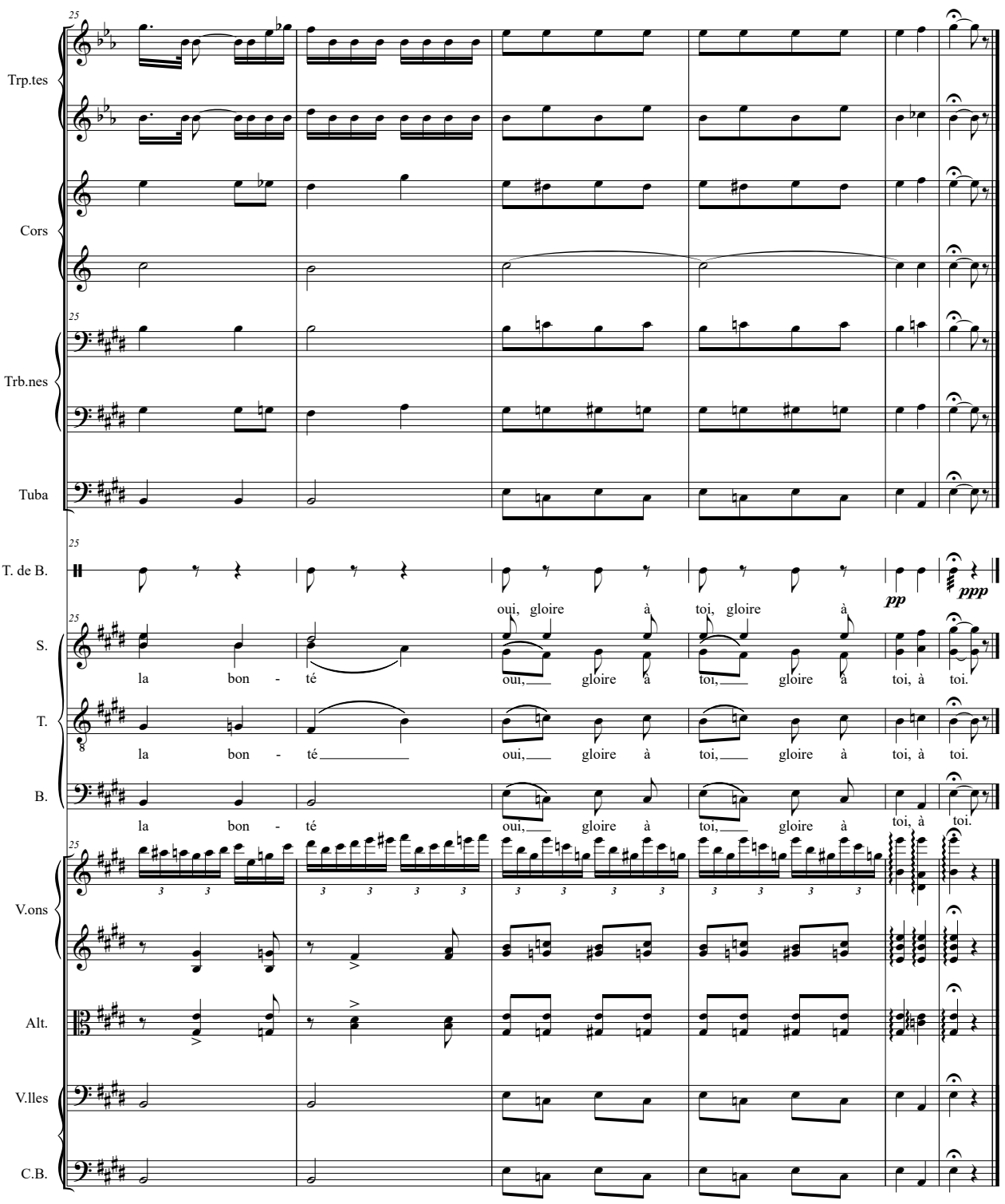

$\mathrm{N}^{\circ} 6$ de suite quelques accords puis prenez l'Air de la Tosca $\left(\mathrm{n}^{\circ} 7\right)$. 
Réplique:

[Paisiello:] Oui, charmante. [(

a ses nerfs.

Scarpia: Un peu.
Paisiello:] À nous, messieurs[!]

$\mathrm{N}^{\circ} 7$

[2 e acte]

Air de la Tosca
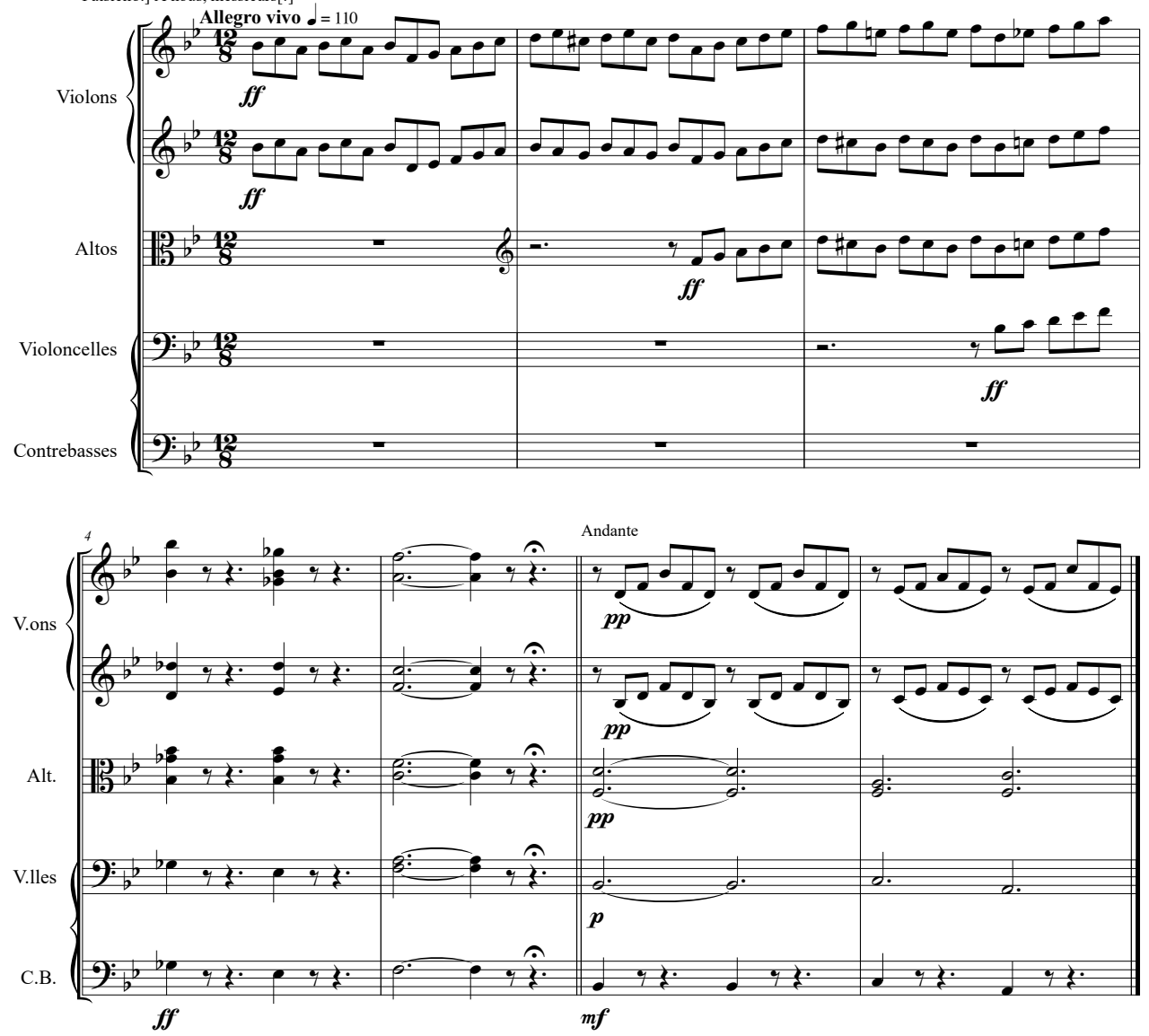

Arrêtez suivant le mouvement de la scène

et finir à la réplique:

[Paisiello:] Basta![...

La musique s'arrête court.] 
Le Musiche di SCENA di Louis Pister Per La Tosca di SARdou: edizione CRITICA
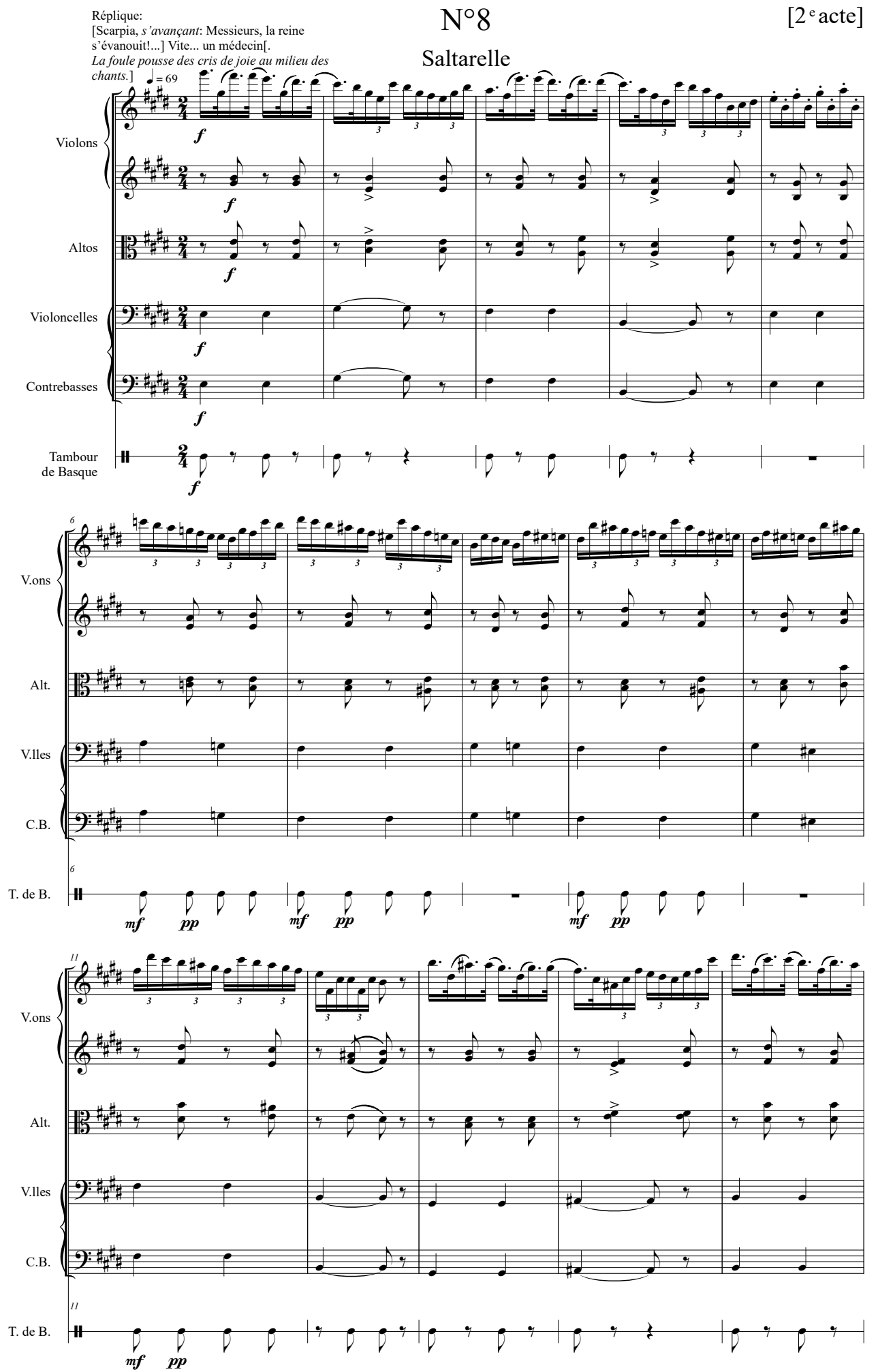

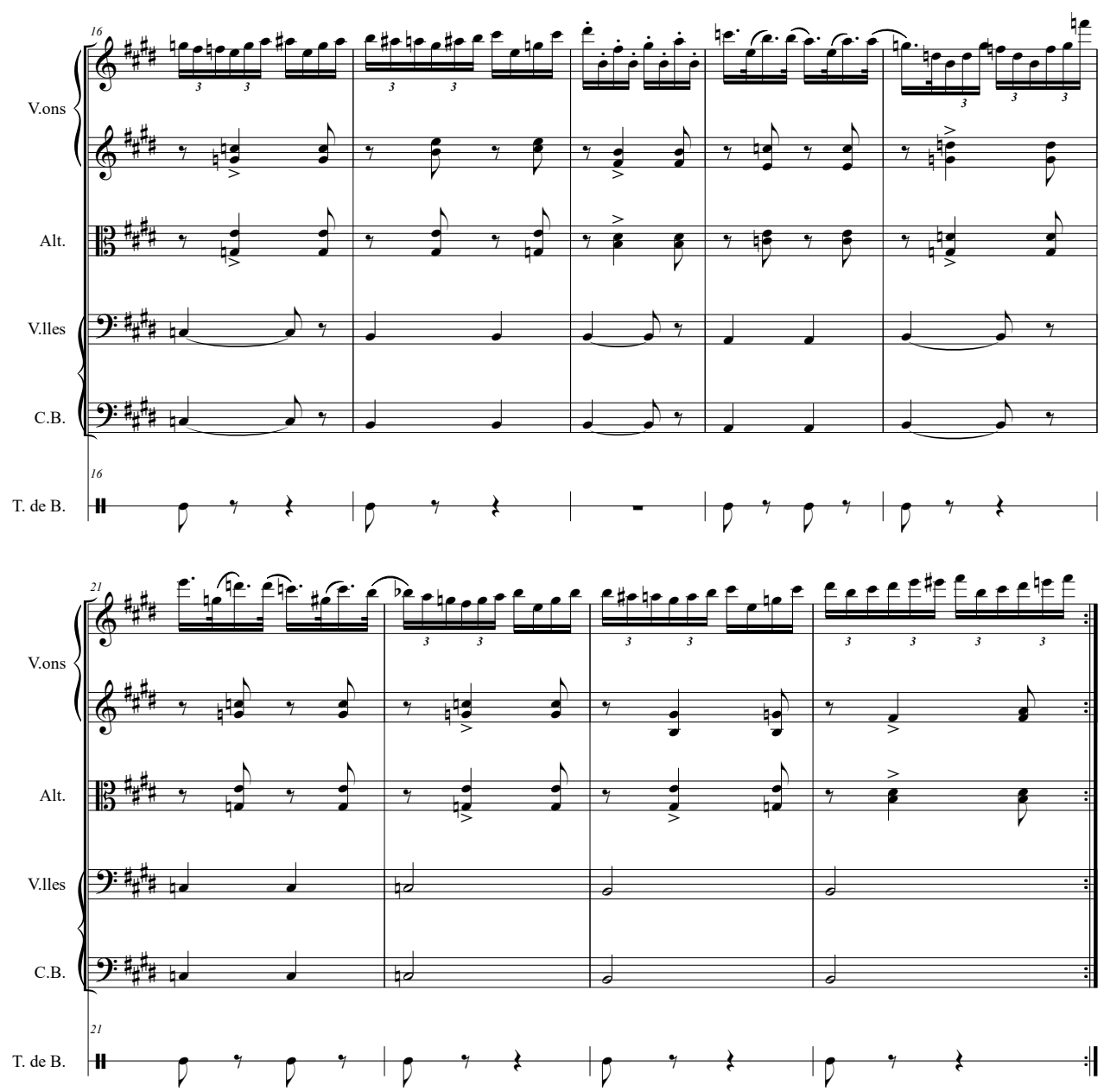

La Saltarelle jusqu'au baisser du rideau[.] 


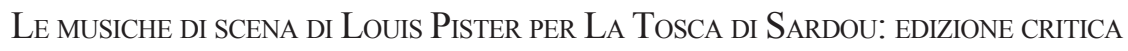

Réplique:

[Floria:] [...] Et c'est devant ça que tremblait toute

une ville[!

$\mathrm{N}^{\circ} 1$

$4^{\mathrm{e}}$ acte

(Roulement de tambour lointain. Trompettes sonnan

la diane. Tressaillant.)]

[Diane]
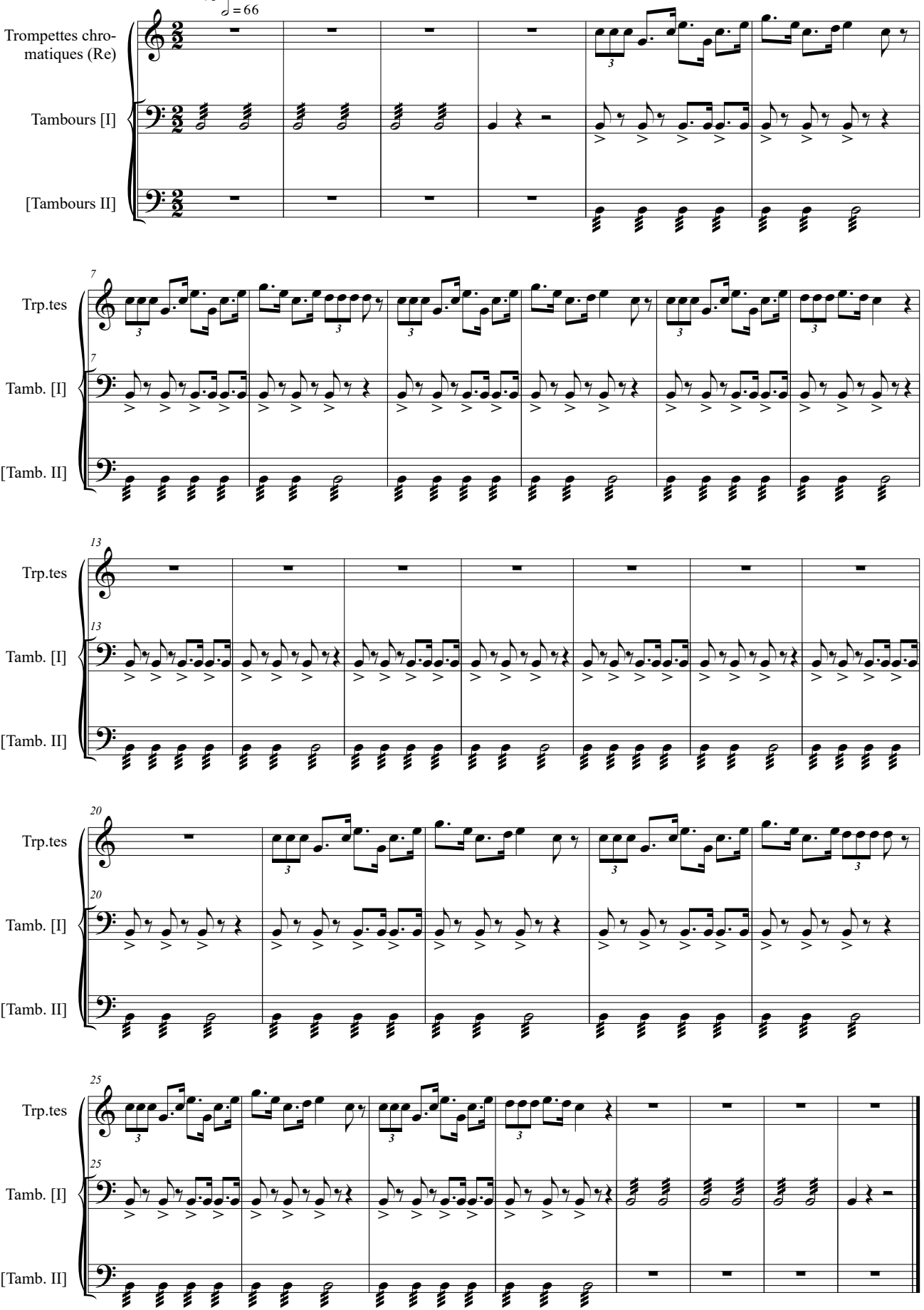

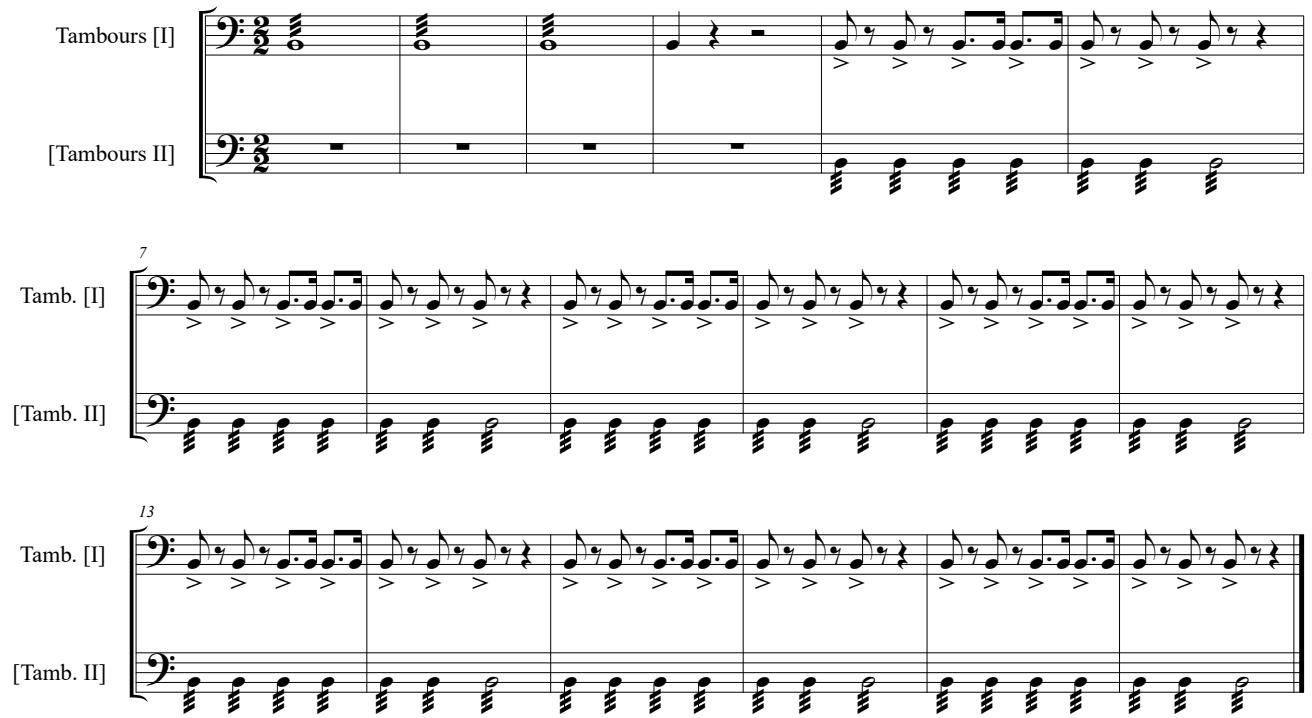

Continuer jusqu'au baisser du rideau[.] 


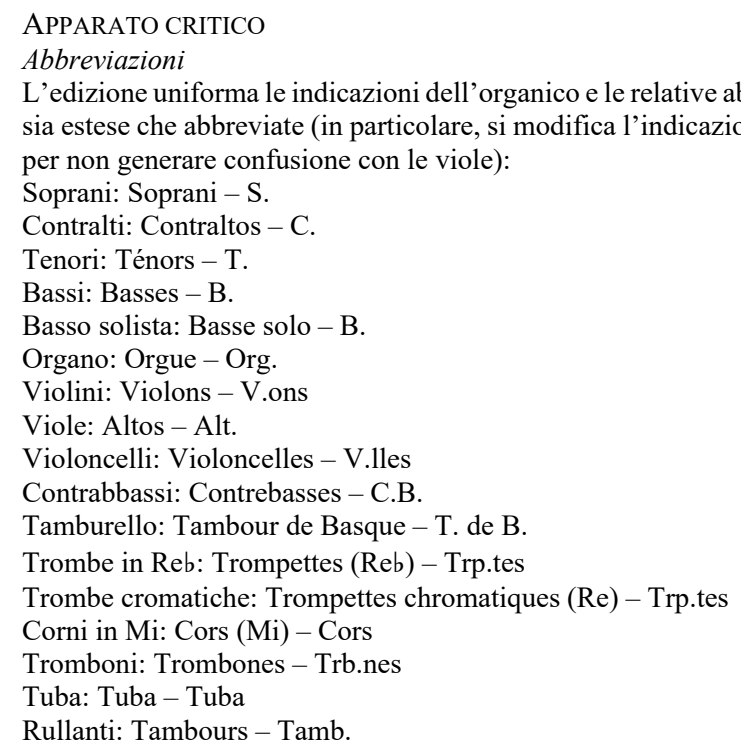

Indicazioni generali

Si emenda il testo delle didascalie a partire dall'edizione critica del testo di Sardou, aggiungendo o emendando quanto attestato in AUS-CAnl MUS JWC 340/1; si sciolgono le ripetizioni.

L'aggiunta od omissione di legature, segni di articolazione (staccati, accenti, corone, arpeggi), indicazioni dinamiche ( $p p, p, m f, f, f f, s f, s f z$, cresc., forcelle) e indicazioni tecniche (arco, pizz.) è condotta sulla base del criterio dell'analogia, a volte con altre voci nello stesso punto, altre volte con altri punti dello stesso brano che presentano una figura musicale simile; per quanto riguarda i tre saltarelli del secondo atto $\left(\mathrm{N}^{\circ} 3, \mathrm{~N}^{\circ} 5 \mathrm{e}^{\circ} 8\right)$, il criterio dell'analogia è ampliato, prendendo in considerazione gli stessi punti nei tre diversi brani per aggiungere od omettere i segni sopra citati; infine, rispetto al minuetto del secondo atto, l'analogia parte anche dal confronto con la fonte F-Pn VM12-23193. Nei casi in cui si rendano necessarie emendazioni o aggiunte per le quali non sussista alcun dubbio (ad esempio quando tutte le voci presentino un'indicazione dinamica tranne una, oppure quando una medesima figura musicale sia attestata due volte, prima con un'articolazione $\mathrm{e}$ poi senza), esse sono condotte senza che l'indicazione o l'articolazione venga aggiunta tra parentesi.

Psaume à 4 voix

48 Org.:
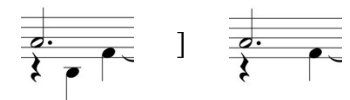

AUS-CAnl MUS JWC 340/1.

Menuet

0 V.ons I: $m f$ ] $p$ F-Pn VM12-23193.

12, 14 V.lles: si omette la legatura tra tutte le note attestata in F-Pn VM12-23193, contrastante con l'indicazione pizz.

27 V.ons I:

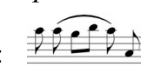

AUS-CAnl MUS JWC 340/1.

32 Alt:

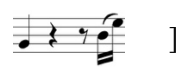

37 V.ons II:

39 V.ons II:

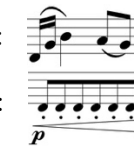

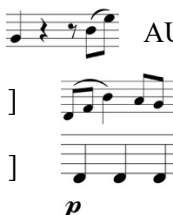

$\boldsymbol{p}$
AUS-CAnl MUS JWC 340/1.

AUS-CAnl MUS JWC 340/1.

AUS-CAnl MUS JWC 340/1. 


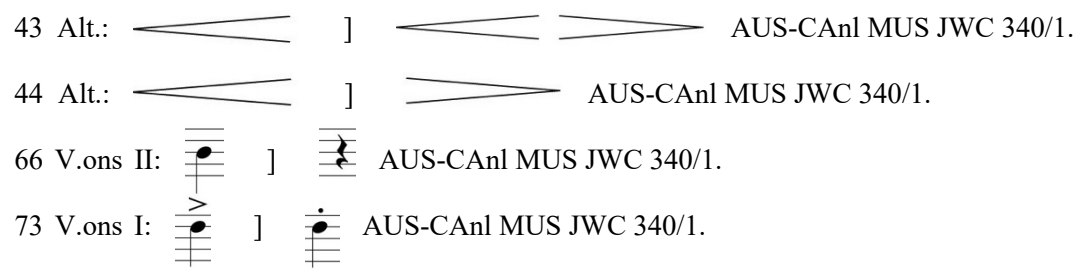

Saltarelle Chour et Quatuor

4 V.ons I: fa\# $\left.\#_{4}\right]$ sol\# $\#_{4}$ AUS-CAnl MUS JWC 340/1.

14 V.ons I: fa\# ${ }_{3}$ ] mi $\#_{3}$ AUS-CAnl MUS JWC 340/1.

21 B.: $\mathrm{do}_{2}$ ] do\#2 AUS-CAnl MUS JWC 340/1.

Andante

6 V.ons I: $]=$ AUS-CAnl MUS JWC 340/1.

26 V.ons I: $p$ ] pp AUS-CAnl MUS JWC 340/1.

Saltarelle/Fanfare Chour et Quatuor

4 V.ons II: $\mathrm{si}_{3}$ ] la 3 AUS-CAnl MUS JWC 340/1.

5 V.lles, C.B.: fa\# ${ }_{2}$ ] sol\# ${ }_{2}$ AUS-CAnl MUS JWC 340/1.

11 V.ons II: fa\# ${ }_{3}-$ do $_{4}$ ] la ${ }_{3}$ AUS-CAnl MUS JWC 340/1.

12 V.ons II: re\# ${ }_{3}-\mathrm{Si}_{3}$ ] $\mathrm{mi}_{3}-\mathrm{Si}_{3}$ AUS-CAnl MUS JWC 340/1.

19 V.ons I: la\#4 la la $_{4}$ AUS-CAnl MUS JWC 340/1.

22 V.ons I: fa5 ] re\#5 AUS-CAnl MUS JWC 340/1.

23 B.: $\mathrm{do}_{2}$ ] do\# $\#_{2}$ AUS-CAnl MUS JWC 340/1.

25 V.ons I: $\mathrm{sol}_{4}$ ] sol\# 4 AUS-CAnl MUS JWC 340/1.

29 T. de B.: $p p$ ] $p$ AUS-CAnl MUS JWC 340/1.

\section{Saltarelle}

6 V.ons I: $\mathrm{mi}_{4}$ ] re\# ${ }_{4}$ AUS-CAnl MUS JWC 340/1.

7, 9, 11 T. de B.: $p p$ ] ppp AUS-CAnl MUS JWC 340/1.

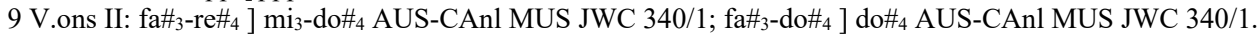

10 V.ons II: $\mathrm{reH}_{3}-\mathrm{Si}_{3}$ ] $\mathrm{mi}_{3}-\mathrm{Si}_{3}$ AUS-CAnl MUS JWC 340/1.

17 V.ons I: la $\left.\#_{4}\right] \mathrm{la}_{4}$ AUS-CAnl MUS JWC 340/1.

20 V.ons I: fa $\mathrm{f}_{5}$ ] fa\#t 5 AUS-CAnl MUS JWC 340/1. 